University of Nebraska - Lincoln

DigitalCommons@University of Nebraska - Lincoln

Timothy J. Gay Publications

Research Papers in Physics and Astronomy

January 1992

\title{
Extrapolation procedures in Mott electron polarimetry
}

Timothy J. Gay

University of Nebraska - Lincoln, tgay1@unl.edu

M. A. Khakoo

University of Missouri-Rolla, Rolla, Missouri

J. A. Brand

University of Missouri-Rolla, Rolla, Missouri

J. E. Furst

University of Missouri-Rolla, Rolla, Missouri

W. V. Meyer

University of Missouri-Rolla, Rolla, Missouri

See next page for additional authors

Follow this and additional works at: https://digitalcommons.unl.edu/physicsgay

Part of the Physics Commons

Gay, Timothy J. ; Khakoo, M. A.; Brand, J. A.; Furst, J. E.; Meyer, W. V.; Wijayaratna, W. M. K. P.; and Dunning, F. B., "Extrapolation procedures in Mott electron polarimetry" (1992). Timothy J. Gay Publications. 37. https://digitalcommons.unl.edu/physicsgay/37

This Article is brought to you for free and open access by the Research Papers in Physics and Astronomy at DigitalCommons@University of Nebraska - Lincoln. It has been accepted for inclusion in Timothy J. Gay Publications by an authorized administrator of DigitalCommons@University of Nebraska - Lincoln. 
Authors

Timothy J. Gay, M. A. Khakoo, J. A. Brand, J. E. Furst, W. V. Meyer, W. M. K. P. Wijayaratna, and F. B. Dunning 


\title{
Extrapolation procedures in Mott electron polarimetry
}

\author{
T. J. Gay, M. A. Khakoo, a) J. A. Brand, J. E. Furst, W. V. Meyer, ${ }^{\text {b) }}$ \\ and W. M. K. P. Wijayaratna \\ Laboratory for Atomic and Molecular Research and Physics Department, University of Missouri-Rolla, \\ Rolla, Missouri 65401 \\ F. B. Dunning \\ Department of Space Physics and Astronomy and the Rice Quantum Institute, Rice University, Houston, \\ Texas $7725 I$
}

(Received 7 August 1991; accepted for publication 20 September 1991)

\begin{abstract}
In standard Mott electron polarimetry using thin gold film targets, extrapolation procedures must be used to reduce the experimentally measured asymmetries $A$ to the values they would have for scattering from single atoms. These extrapolations involve the dependence of $A$ on either the gold film thickness $t$ or the maximum detected electron energy loss in the target $\Delta E$. Using a concentric cylindrical-electrode Mott polarimeter, we have studied and compared these two types of extrapolations over the electron energy range 20-100 keV.

The potential systematic errors which can result from such procedures are analyzed in detail, particularly with regard to the use of various fitting functions in thickness extrapolations, and the failure of perfect energy-loss discrimination to yield accurate polarizations when thick foils are used. A critical discussion of previous work on this subject is presented.
\end{abstract}

\section{INTRODUCTION}

Electron spin polarizations are frequently measured using Mott scattering from thin gold films. ${ }^{1-3}$ If a beam of electrons is scattered by the gold target into two detectors arranged at equal polar angles and opposite azimuthal angles relative to the beam direction, then the electron polarization transverse to the scattering plane $P$ is given in an ideal experiment by

$$
P=A / S_{\text {eff }}
$$

where

$$
P=\frac{N_{1}-N_{1}}{N_{1}+N_{1}}
$$

and $N_{\mathrm{t}}$ and $N_{\mathrm{t}}$ are the beam intensities with spin-up and spin-down relative to an axis of quantization perpendicular to the scattering plane. The "measured scattering asymmetry" is defined to be

$$
A \equiv \frac{L-R}{L+R}
$$

where $L$ and $R$ are the number of electrons detected in the "left" and "right" counters, respectively. The analyzing power of the apparatus, $S_{\text {eff }}$ is often called the "effective Sherman function," and must be known in order to determine $P$ from a measured value of $A$.

The value of $S_{\text {eff }}$ can be determined in a number of ways. One method involves a double-scattering experiment in which initially unpolarizcd electrons scatter from two equivalent targets, and $S_{\text {eff }}$ is measured directly. ${ }^{4,5}$ A second technique uses beams of electrons with known polar-

\footnotetext{
a) Permanent address: Physics Department, California State UniversityFullerton, Fullerton, CA 92634-9480.

${ }^{b}$ Present address: NASA Lewis Research Center, Cleveland, OH 44135.
}

ization to calibrate the Mott analyzer. ${ }^{6-9}$ We consider here a third method which is the simplest to carry out experimentally, and which is by far the most common. Essentially, it involves the measurement of $A$ for experimental conditions corresponding as closely as possible to elastic scattering from single $\mathrm{Au}$ atoms. In this case, the Mott analyzing power $S$ can be calculated theoretically, and $S_{\text {eff }}=S$.

Attempts to meet the criteria of elastic, single scattering experimentally have used either electron energy-loss extrapolations or target-thickness extrapolations. In the "standard" Mott polarimeter, scattered electrons are monitored by scintillators or surface-barrier detectors at the same electrostatic potential as the target foil. They are thus detected with energy resolutions characteristic of these devices-about $10 \mathrm{keV}$. To determine the experimental asymmetry which can be associated with single (albeit possibly inelastic) scattering conditions, $A$ is measured for a range of film thickness $t$, and then extrapolated linearly to zero thickness where this condition must, by definition, be met. With the advent of concentric-electrode polarimeters of the Farago/Rice design, a different type of extrapolation procedure has become possible. ${ }^{1-3,7,10-14}$ Such devices have, in principle, arbitrarily good energy resolution, allowing the measurement of $A$ as a function of $\Delta E$, the maximum energy which an electron can have lost in the target and still be detected. The value of $\Delta E$ is determined by a retarding potential placed on apertures in front of the electron-multiplier detectors, or on the front surface of the detectors themselves. Thus a value of $A$ can be measured which corresponds to purely elastic (albeit possibly multiple) scattering of electrons by the target.

In this article, we consider in detail systematic errors in the determination of $P$ which can occur in polarimetric measurements as a result of experimental extrapolations attempting to ensure "atomic scattering" conditions. In the 


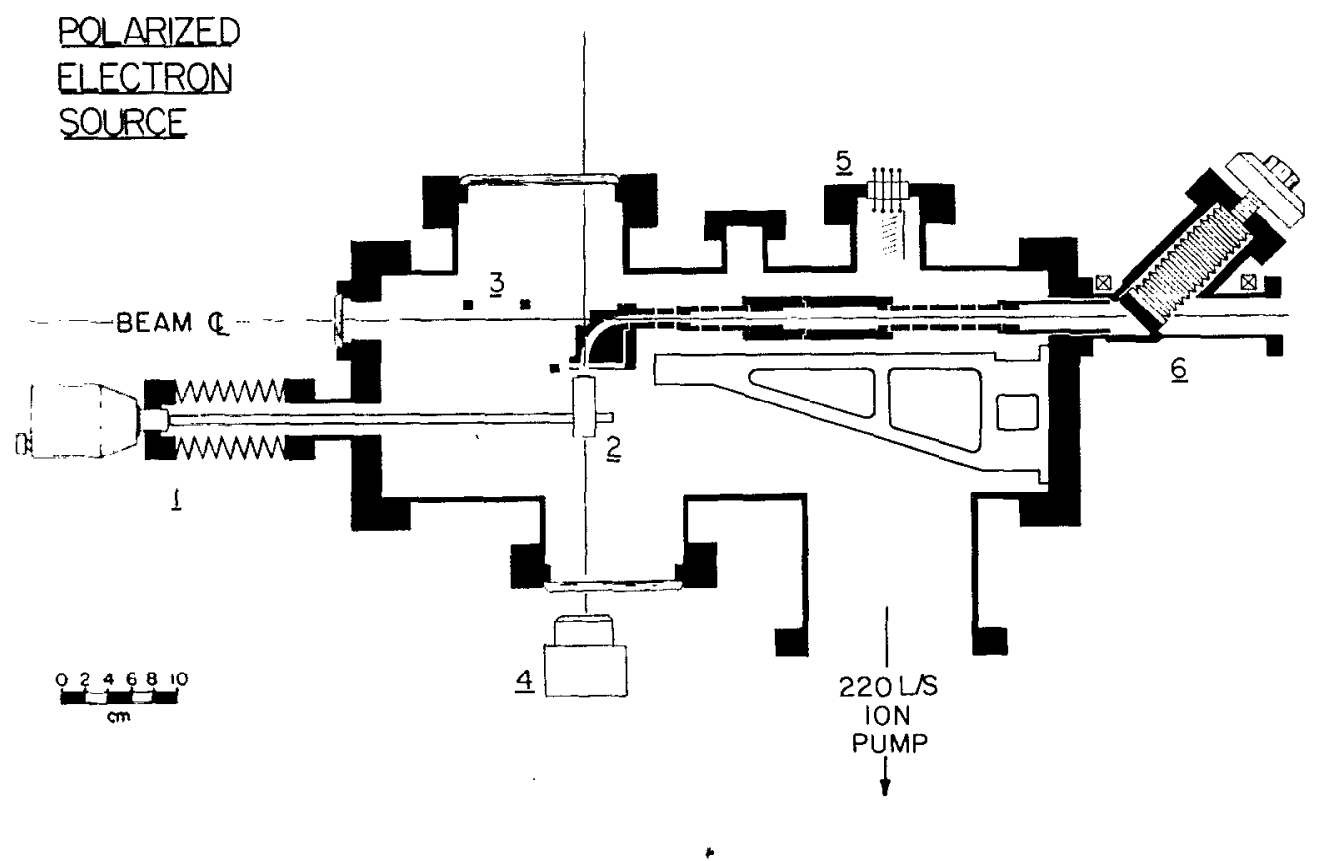

FIG. 1. Scale drawing of polarized electron source: (1) rotary and linear motion feedthrough for manipulation of the GaAs photocathode; (2) mount and heater for the GaAs wafer; (3) cesiator, represented by black solid squares-the two at the left are used for crystal activation, the one at the right is used for continuous cesiation while electrons are extracted; (4) GaAlAs laser power meter; (5) nude ion gauge; (6) bakeable inline isolation valve (GranvillePhillips model 209) with magnetic steering coil.

experiments reported here, beams of polarized electrons were produced with a GaAs photoemission source, and their transverse polarization $P$ was measured using a concentric-cylindrical-electrode Mott polarimeter. A systematic study was made of the dependence of $A$ [Eq. (3)] on $t$, $\Delta E$, and $E$, the kinetic energy of the electrons just before scattering from the Mott target. The total detected electron signal, $N=L+R$, was also studied as a function of these parameters. Systematic studies of this type have been carried out previously. ${ }^{7,10-12,15}$ and we view this report as an extension and analysis of those investigations.

As mentioned before, $S$ is calculated assuming elastic scattering from single atoms. While thickness extrapolations made with sufficiently thin targets ensure that $A$ can be associated with single-atom scattering, and $\Delta E$ extrapolations may ensure elasticity, it is apparent that neither a thickness extrapolation with poor energy resolution (as is done in "standard" Mott experiments), nor a $\Delta E$ extrapolation using a target of finite thickness can guarantee that both of these conditions are met. Indeed, at some (perhaps negligibly low) level, systematic error must result from either of these procedures. Only in the limit as $t$ and $\Delta E$ approach zero simultaneously can the theoretically assumcd conditions be cnsured.

The ultimate accuracy of Mott measurements of the type considered here (as opposed to double scattering or externally calibrated measurements) depends on three factors: the accuracy of the theoretical calculations of $S$, the degree to which "apparatus" errors such as geometric misalignments and beam instabilities can be eliminated (see Sec. III A), and the extent of systematic errors due to target extrapolation procedures. In this article we address exclusively the latter issue, but remind the reader that, in general, the first two factors can be comparably important in limiting accuracy.

\section{POLARIZED ELECTRON SOURCE AND MOTT POLARIMETER}

\section{A. Source}

A scale drawing of the polarized elcetron source is shown in Fig. 1. Its design has been strongly influenced by the reports of the CCNY ${ }^{16,17}$ and NIST groups. ${ }^{18}$ The source vacuum chamber is ultrahigh vacuum (UHV) compatible and is evacuated with $220 \mathrm{\ell} / \mathrm{s}$ diode noble-gas ion pump. Its base pressure after a one-week bake at $250{ }^{\circ} \mathrm{C}$ is $\sim 4 \times 10^{-11}$ Torr, although this deteriorates over a period of months to $\sim 5 \times 10^{-10}$ Torr due to source operational procedures, including heating, cesiation, and oxygenation of the GaAs crystals.

Two rectangular $\mathrm{GaAs}\langle 110\rangle$ wafers $^{19}$ of approximate dimension $8 \mathrm{~mm} \times 20 \mathrm{~mm}$ are mounted in a holder which can be translated and rotated precisely. ${ }^{20}$ The wafers are cleaned chemically prior to installation in the vacuum chamber using a procedure essentially identical to that described in Ref. 16. Once at UHV, they are prepared for use by resistive heat cleaning, ${ }^{21}$ followed by cesiation and oxygenation, again in a manner similar to that of Ref. 16 . Activated crystals are moved from the preparation position to a position directly under the $90^{\circ}$ electrostatic bend. There, circularly polarized light at $792 \mathrm{~nm}$ from a GaAlAs diode laser ${ }^{22}$ strikes them at normal incidence with $\mathrm{a} \sim 1$ $\mathrm{mm}$-diam spot size, resulting in the photoemission of longitudinally polarized electrons. ${ }^{18,23}$ For $20 \mathrm{~mW}$ of light incident on the GaAs, we typically observe total photoemission currents of $400 \mu \mathrm{A}$, corresponding to a quantum efficiency of $\sim 3.2 \%$. While the crystal is under the electrostatic bend, its surface is continuously cesiated at a low level by a Cs dispenser ${ }^{24}$ near the edge of, and just under, the first extraction electrode. This Cs replaces that which over time desorbs from the GaAs, and produces a very stable negative electron affinity surface, which in turn 
yields stable electron-beam currents and effectively infinite source lifetimes.

The $90^{\circ}$ electrostatic bend converts the longitudinal electron polarization into transverse polarization. Prior to exiting the source chamber, the beam is collimated and focused by a series of cylindrical lenses and apertures. The Mott polarimeter and source are connected by a vacuum pipe containing an inline bakeable valve and a collimating aperture of 1/8-in. diameter. Beam transport through this pipe can occur at ground potential because the GaAs photocathode is maintained at $-2 \mathrm{kV}$. This large negative potential produces a very "stiff" beam that is easily directed into the Mott analyzer. Two sets of magnetic-dipole steering doublets and an axial, solenoidal spin rotator are mounted on this tube as well.

\section{B. Polarimeter}

Scale diagrams of the Mott polarimeter are shown in Figs. 2 and 3. The polarimeter is a cylindrical, retardingfield device of the Farago/Rice type, ${ }^{10,11,14}$ and is completely UHV compatible. It is pumped by a $110 \mathrm{l} / \mathrm{s}$ diode noble-gas ion pump and has a base pressure of $\sim 2 \times 10^{-9}$ Torr which is limited, we believe, by the porous ceramic of the high voltage bushing and the presence of Formvar, used as a target backing, in the chamber. A highly polished stainless-steel inner cylinder is maintained at a positive voltage relative to ground of between 20 and $125 \mathrm{kV}$. The inner cylinder is electrically isolated from ground by a ceramic break, atop which is mounted a linear-motion feedthrough that enables target foils of varying thickness to be moved onto the beam line while the cylinder is at high voltage.

Electrons accelerated in the radial field between the inner and grounded outer cylinders collide with one of the target foils, and may be scattered by $120^{\circ}$ to either the left or right in the plane of the diagram in Fig. 3. If they have lost less than $2 \mathrm{keV}$ in the foil, they can pass through an aperture in the outer cylinder and enter the grounded entrance aperture of one of the retarding-field analyzers (RFAs; Fig. 4). The RFAs comprise a grounded entrance shield, a double-aperture retarding-field electrode, a continuous-dynode electron multiplier (CDEM), and a cylindrical electron shield. The maximum energy loss in the foil of the detected electrons $\Delta E$ is determined by setting $V_{r}$ the potential on the retarding apertures: $\Delta E=2 \mathrm{keV}-V_{r}$ A more detailed description of the retarding field analyzers will be given in the next section.

Electrons which pass through the target foil and the exit aperture of the inner cylinder are decelerated and leave the Mott chamber through an enlarged hole in the outer cylinder. (The diameter of this hole has been increased to reduce the number of secondary or reflected electrons, resulting from the primary beam striking the outer cylinder, which could be reaccelerated back through the target and scattered through $60^{\circ}$ into the detectors.) They are then refocused and either enter a Faraday cup or strike a fluorescent $\mathrm{ZnS}$ screen which serves as a beam-profile monitor.

Because of several issues that will be raised in Sec. IV, we discuss now, in some detail, our Mott scattering targets.

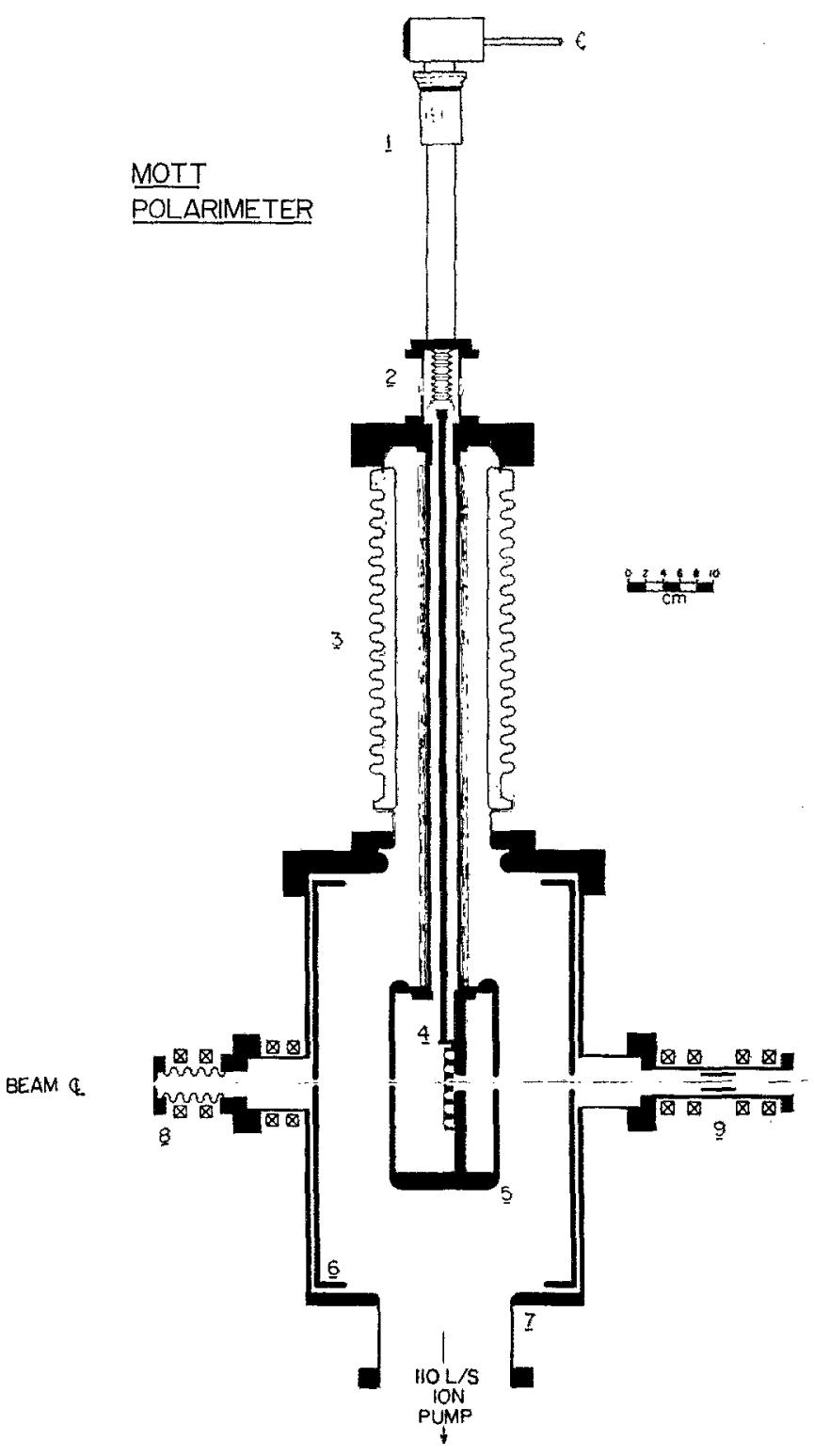

FIG. 2. Scale side sectional view of the Mott polarimeter: (1) mitre gear assembly and linear motion feedthrough for movement of target foils at high voltage; (2) glass break isolating target holder assembly from inner cylindrical electrode, allowing target foils to be negatively biased for desorbed ion suppression; (3) high voltage bushing (Ceramaseal special) the inner support cylinder is surrounded by a Pyrex tube to reduce the possibility of electrical breakdown; (4) target holder assembly (ceramic standoffs from inner cylinder not shown); (5) inner cylindrical electrode; (6) outer cylinder held at ground potential; (7) vacuum chamber; (8) entrance aperture with magnetic-dipole steering doublets; (9) exit steering and focusing assembly prior to Faraday cup.

A series of five target films was made by vacuum evaporation of high-purity gold wire onto Formvar ${ }^{25}$ foils. In order to approach the single-atom scattering case as closely as possible, we made targets which include the thinnest $\mathrm{Au}$ films used in Mott polarimetry to date. Previously, the thinnest films used had densities of 16 (Ref. 26) and 19 (Refs. 7, 11,27) $\mu \mathrm{g} / \mathrm{cm}^{2}$. Our Au film areal densities were determined by $\mathrm{x}$-ray fluorescence (XRF) measurements to be $6.6,16.5,32.9,65.8$, and $132 \mu \mathrm{g} / \mathrm{cm}^{2}$, corresponding to average thicknesses between 34 and $682 \AA$. These values, 


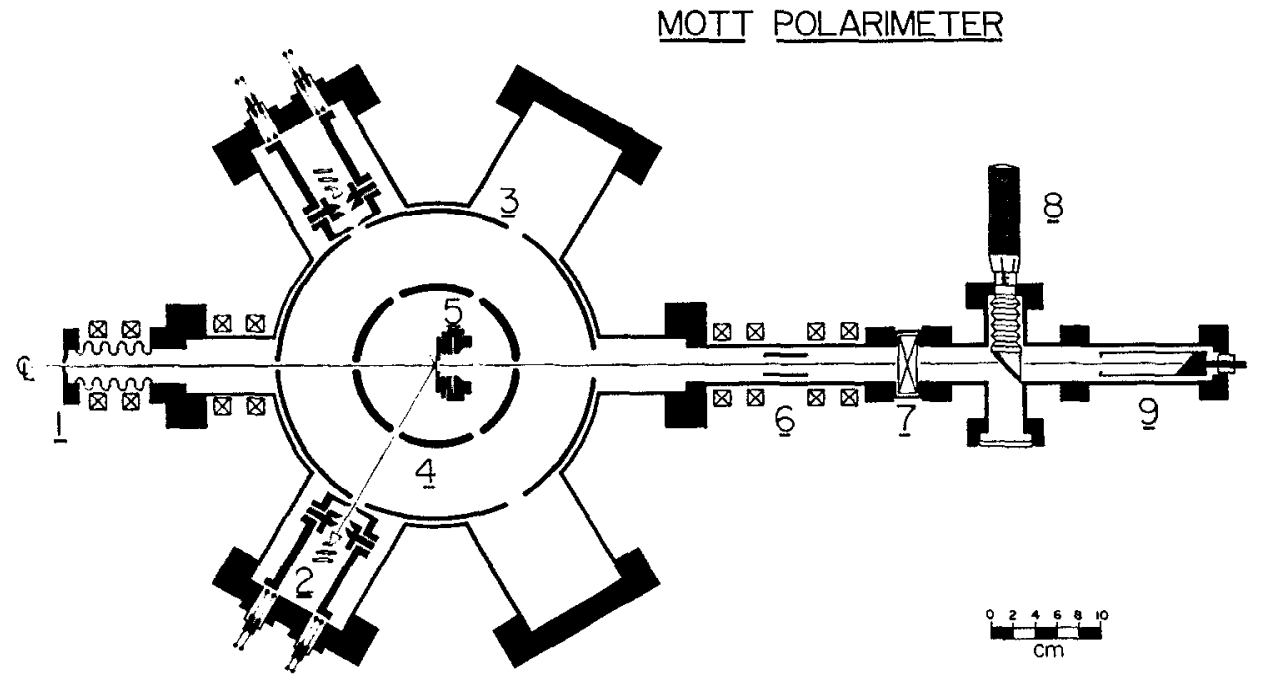

FIG. 3. Scale top sectional view of the Mott polarimeter: (1) entrance aperture and magnetic steering elements; (2) retarding field analyzer with continuous-dynode electron multiplier (see Fig. 4) and electrical feedthroughs; (3) outer cylinder; (4) inner cylinder; (5) target holder; (6) exit focusing and steering assembly; (7) gate valve; ( 8 ) retractable $\mathrm{ZnS}$ beam viewing screen; (9) Faraday cup.

which have an absolute uncertainty of about $25 \%$, have relative uncertainties of $10 \%$, except for the thinnest target, whose thickness has a relative uncertainty closer to $15 \%$. Estimates of these latter uncertainties are based on experiments which will be discussed in Sec. IV.

The morphology of our target films varies over their areal density range. All of the Formvar backings and $\mathrm{Au}$ films appeared taut and flat to the eye. X-ray depth profiling showed that on a $100-\mu \mathrm{m}$ scale, target areal densities were very uniform. On a $0.1-\mu \mathrm{m}$ scale, however, the four thinnest films exhibited nonuniformities due to the formation of nucleated droplets. This is a well known phenomenon which occurs in extremely thin films when the film material does not wet the substrate. ${ }^{28,29}$ To study the target structure in more detail, we made transmission electron microscopy (TEM) micrographs of our films. For the 6.6$\mu \mathrm{g}$ target, well separated droplets were spread uniformly across the Formvar surface. As Au areal density increased,

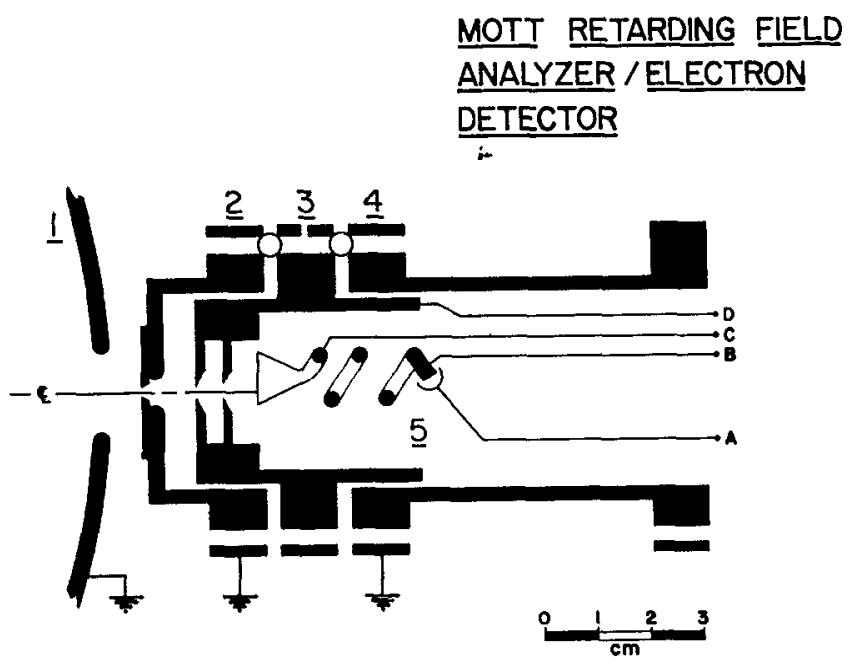

FIG. 4. Scale sectional view of a retarding-field analyzer/electron detector: (1) outer cylinder of Mott polarimeter; (2) entrance aperture; (3) retarding potential electrode held at $V_{r}(D) ;(4)$ background electron shield; (5) continuous-dynode electron multiplier with potentials $V_{r}+135 \mathrm{~V},(C), V_{r}+2135 \mathrm{~V},(B)$, and $V_{r}+2235 \mathrm{~V},(A)$. these drops coalesced and were largely contiguous in the $66-\mu \mathrm{g} / \mathrm{cm}^{2}$ sample. The $132 \mu \mathrm{g} / \mathrm{cm}^{2}$ film appears to be uniform. Using a gray-scale computer analysis of the TEM scans, we estimate the Au coverage to be $38 \%, 58 \%, 73 \%$, $89 \%$, and $100 \%$, ranging from the 6.6 to the $132 \mu \mathrm{g} / \mathrm{cm}^{2}$ sample. The effects of target morphology on the precision of our measurements will be discussed in Sec. IV.

While concentric-electrode polarimeters have a number of advantages over "standard" Mott polarimeters, ${ }^{3}$ two of them are particularly relevant for the studies reported here. The first, of course, is that they allow $\Delta E$ extrapolations to be made at all. Elastic scattering is assumed in theoretical calculations of $S$, and the use of the concentric electrode design allows us to meet this condition experimentally. Moreover, the cylindrical geometry is convenient for the placement of a number of target foils in the vacuum at once, making simultaneous $t$ and $\Delta E$ extrapolation possible. Second, the pernicious problem of wall- and slit-scattered background ${ }^{1,3}$ is significantly reduced. Such secondary scattering events are usually associated with significant energy loss. ${ }^{30}$ They can thus be effectively discriminated against as $\Delta E$ is reduced to zero.

\section{DATA ACQUISITION AND ANALYSIS}

\section{A. Correction for instrumental asymmetries}

Detector counts were determined as a function of four parameters: $\Delta E, E$, nominal target thickness $t$, and electron spin direction. At a given $E, t$ and $\Delta E$, the experimental asymmetry was measured for "spin-up" and "spin-down" beams to correct for the effects of instrumental asymmetries. Such asymmetries, corresponding to lack of geometric or electronic equivalence between the two detectors, result, for example, in a nonzero value of $A$ [Eq. (3)] for unpolarized incident electrons. The change of electron-spin orientation was accomplished by optically flipping the helicity of the photons used to produce photoemission from the GaAs source crystal. ${ }^{16-18}$ By assuming that there are no electron-spin-correlated beam trajectory effects, and that the spin is accurately reversed, it can be shown ${ }^{1-3,11}$ that 
first-order corrections for geometric misalignments yield a true Mott asymmetry

$$
A=\frac{\Delta-1}{\Delta+1}
$$

where

$$
\Delta=\left(\frac{(L \uparrow)(R \downarrow)}{(L \downarrow)(R \uparrow)}\right)^{1 / 2}
$$

and the (background-corrected) count rates given in parentheses in Eq. (5) are for the "left" and "right" detectors with spin-up and spin-down beams.

Use of Eqs. (4) and (5) does not eliminate the possibility of error in $A$ due to second-order instrumental asymmetry effects or abrupt changes in measurement conditions (such as beam current changes) on a time scale comparable to data accumulation intervals. ${ }^{1-3}$ Our raw data routinely exhibited instrumental asymmetries, defined experimentally as

$$
A_{I}=\frac{1-\Delta_{I}}{1+\Delta_{I}}
$$

where

$$
\Delta_{I}=\left(\frac{(L \uparrow)(L \downarrow)}{(R \uparrow)(R \downarrow)}\right)^{1 / 2} .
$$

Values of $A_{I}$ varied from run to run, but were typically between 0.2 and 0.4 , corresponding to about a $2: 1$ countrate ratio between the two detectors. In extreme cases, $A_{I}$ was as high as 0.6. We thus expect that some residual second-order effects due to instrumental asymmetries may affect our data, although not at the level of the uncertainties which we quote. Evidence for this assertion comes from the fact that even when $A_{I}$ 's exhibited large variations as a result of changes in the beam energy or RFA operating parameters, corresponding variations in $A$ were not observed.

While the relative precision of our data thus appears to be unaffected by these sccond-order effects, its absolute accuracy is uncertain because we could not completely test the assumptions which render Eqs. (4) and (5) valid to first order - that optical reversal of the electron spins was complete and that there were no spin-related beam trajectory changes (see, however Sec. III D below). Ultimately, a complete characterization of instrumental asymmetries and the subsequent absolute accuracy of $P$ can be accomplished only with the careful use of monitor counters, preferably in combination with low- $Z$ foils. ${ }^{1-5}$ Because our present apparatus did not incorporate these features, we wish to emphasize that the uncertainties which we quote are for the relative precision of our data, and not its absolute accuracy.

\section{B. Variation of $\Delta E$-Retarding-field spectra}

While the variation of $t$ is straightforward for a given $E$, we encountered considerable difficulty in developing RFAs which had good energy resolution and which behaved in an understandable fashion as $\Delta E$ was varied. In
$\mathrm{Au}$, inelastic scattering associated with plasmon production can occur with energy losses as little as $6 \mathrm{eV}^{31,32}$ It was thus considered desirable that the RFA resolution be better than $\sim 5 \mathrm{eV}$ so that elastic scattering could be studied cleanly, and so that, in principle, direct comparisons could be made between our data and the calculated values of $S$ for elastic scattering. By measuring the CDEM count rate as a function of the retarding voltage $V_{n}$ an integral electron energy-loss spectrum is accumulated. ${ }^{33}$ An example of such a spectrum, taken with our apparatus in its final configuration, is shown in Fig. 5(a). It is characterized by a sharp onset of counts near $\Delta E=0$, and is monotonic in $\triangle E$. Retarding-field spectra taken with earlier RFA configurations exhibited broad, shallow $\Delta E=0$ cutoffs indicative of poor energy resolution, local minima characteristic of electron focusing or reflection effects ${ }^{33}$ and/or spurious secondary-electron detection, or both.

The final configuration of our RFAs is shown in Fig. 4. It has a number of features which result in its superior performance. Electrons which have lost less than $2 \mathrm{keV}$ in the target are tightly collimated by a 2.3 -mm-diam grounded entrance aperture cut in a tantalum disk. This collimator serves to limit transverse momenta of the entering electrons with a subsequent improvement in the analyzer's energy resolution. ${ }^{33}$ Moreover, most of the secondary electrons created in the analyzer originate at this first defining aperture, and are subsequently discriminated against in the retarding-field region. The retarding-field region is defined by two additional tantalum disks separated by a $5-\mathrm{mm}$ spacer. The disk closest to the entrance of the RFA has a 2.6-mm-diam aperture; the second has an aperture of $3.2-\mathrm{mm}$ diameter. The size of the holes results from a compromise between maximum retarding-field definition and the reduction of spurious secondary electrons created at aperture knife cdges. The use of two equipotential disks instead of a single retarding electrode provides a more uniform retarding field over a large volume, and is the single most important factor both in eliminating the spectral minima mentioned earlier, and in improving the analyzer's energy resolution. The use of high-transmission grids to define the retarding volume was avoided because of concerns about secondary-electron production. ${ }^{34}$

Electrons which can surmount the retarding potential barrier are immediately accelerated into the entrance cone of the CDEM by a $+135 \mathrm{~V}$ potential difference between the cone and the final retarding-field aperture. This arrangement of voltages, in which the CDEM floats at a constant voltage above the retarding potential, ensures the most uniform possible detection efficiency across the range of electron energies emerging from the retarding-field region. ${ }^{35}$ In the extreme case reported here, for example, in which $\Delta E=1300 \mathrm{eV}$, the electrons which have lost 1300 $\mathrm{eV}$ and can just surmount the retarding potential strike the CDEM with a kinetic energy of $135 \mathrm{eV}$, while the ones which have scattered elastically from the foil have a kinetic energy of $1435 \mathrm{eV}$ at the detector. Over this range, the CDEM's efficiency drops from roughly $97 \%$ to $87 \%$. ${ }^{35}$

The retarding-field spectrum shown in Fig. $5(\mathrm{a})$ has a count rate onset corresponding to an energy resolution, $W$, 


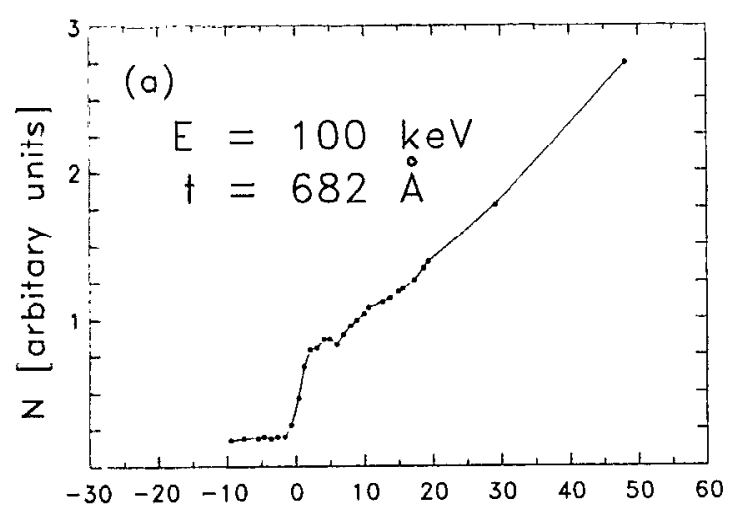

MAXIMUM ELECTRON ENERGY LOSS, $\triangle E(\mathrm{eV})$

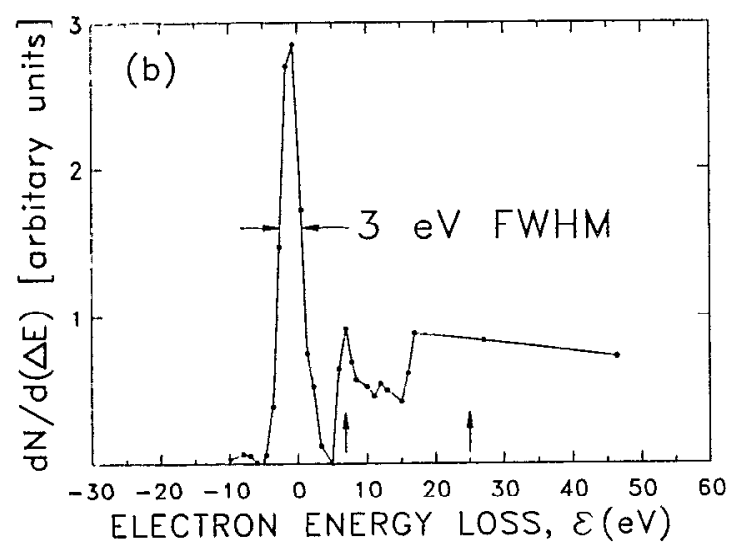

FIG. 5. (a) Retarding-field spectrum; (b) its derivative (smoothed by triple adjacent point averaging) with the $682 \AA$ target at $E=100 \mathrm{keV}$. Major bulk and surface plasmon energies are indicated (see text).

of $<3 \mathrm{eV}$ [full width at half maximum (FWHM)]. In addition, a well defined "plateau" associated with the forbidden energy-loss region between elastic scattering and low-energy plasmon production is apparent. The existence of this plateau means that we are able to unambiguously distinguish elastic scattering from the Mott target. To illustrate this more clearly, the point-to-point difference spectrum (smoothed by triple adjacent point averaging) is shown in Fig. 5(b). The $\sim 3-\mathrm{eV}$-wide elastic peak is well separated from the region of inelastic scattering. Indeed, hints of the major surface and bulk plasmon production features at 7 and $25 \mathrm{eV}$, respectively, can be seen as well. ${ }^{32}$ Thus, our apparatus is capable of energy resolution of the order of three parts in $10^{5}$, a figure comparable to optical monochromators and a few linear accel/decel energy-loss spectrometers. ${ }^{36,37}$ We note that the increase of count rate with increasing $\Delta E$ is not entirely due to an increased number of energy-loss events, but reflects as well the increasing electron-optical solid angle acceptance of the RFAs as $V_{r}$ is lowered. ${ }^{38}$

\section{Background corrections}

The counts accumulated by each detector in a given run were corrected for background from a number of sources. In our preliminary experiments large count rates due to electrons emerging from the ion pump were observed. This problem was eliminated by the insertion of a

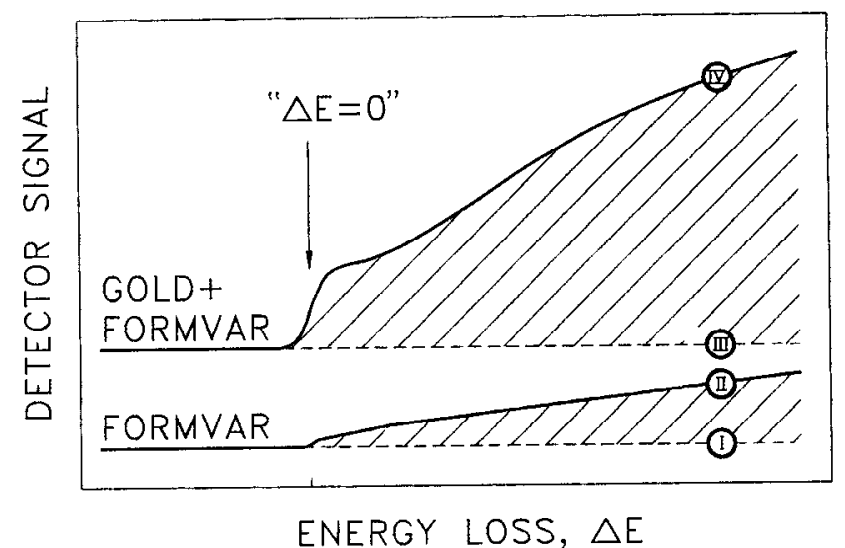

FIG. 6. Schematic retarding-field spectra for $\mathrm{Au}$ and Formvar targets (see text).

one and one-quarter turn stainless-steel "spiral-staircase" baffle between the pump and Mott chamber. The inner cylindrical electrode was carefully polished to minimize corona discharge of electrons, and shields were placed around the CDEMs to reduce the detection of electrons which could find their way into the detector without passing through the retarding field. As a result of these measures, the beam-unrelated count-rate at high voltage, including CDEM dark count (which was $<0.1 \mathrm{~Hz}$ ) was always less than $10 \mathrm{Iz}$.

The remaining background subtraction procedures are best understood by referring to Fig. 6, which shows schematic integral electron energy-loss spectra for both gold and blank Formvar targets. The Formvar backing contribution to gold-target signals must be accurately subtracted, because it has nearly nil real asymmetry, but can represent a significant fraction of the total count rate for the thinnest gold films. (At $100 \mathrm{keV}$ with $\Delta E=1300 \mathrm{eV}$, the Formvar signal was fully half that of the thinnest $\mathrm{Au}$ target. The worst case with elastic scattering and the thinnest target was at $20 \mathrm{keV}$, where the Formvar signal was about $2 \%$ that of the Au.) Both gold and Formvar spectra have components which are independent of $\Delta E$ (indicated as [I] and [III] in Fig. 6), as well as direct target contributions ([II] and [IV]) which for Au targets comes from the Formvar backing in addition to the gold itself. These flat background counts are caused by $\mathrm{x}$ rays due in roughly equal parts to incident electrons striking the target foils and to stray, scattered electrons striking the inner cylinder. The importance of the latter component, which could include desorbed or sputtered ions as well as photons, was verified by allowing the primary beam to pass through the chamber unimpeded, and observing that background counts diminished by about $50 \%$. Ions caused by electron bombardment of the target foils were eliminated by biasing of the foil holders negatively relative to the inner cylinder. ${ }^{11}$ The flat background component of the total signal was small except for the blank Formvar targets, where it was comparable to the elastic signal.

For a given value of $\dot{E}, \Delta E$, and target thickness, the Formvar component of the signal was determined by mea- 
suring count rates from both the Formvar blank and $\mathrm{Au}$ targets, and then determining the $x$-ray background for each target for several values of $V_{r}$ which prevented any scattered electrons from entering the RFAs. The background corrected rate was then taken as (using the notation of Fig. 6) [IV]-[III] minus the pure Formvar signal, [II]-[I]. For a given Au target/Formvar blank run, RFA counts were normalized to the Faraday cup current, with a transmission correction made to compensate for the fact that different scattering in the various targets altered the Faraday cup reading for constant incident current.

\section{General systematic checks}

A number of test experiments were run to check the data and general functioning of the apparatus. The asymmetry was measured for varying beam characteristics by changing focusing and deflector voltages over a wide range. No statistically significant changes in $A$ were found, indicating that the beam is strongly focused and confined to a given point on the target by the radial field between the cylindrical electrodes. This has been found to be the case by other investigators using polarimeters similar to ours, ${ }^{10-12}$ and would indicate that spin correlated trajectory variations of the incident beam, thought to be small to begin with, would have a negligible affect on our data.

We searched for variations in $A$ when a given target film was moved up and down in the beam. No significant dependence on position was observed for any of the targets. This is not surprising, given that the foils appeared quite flat and uniform to the eye over their $1-\mathrm{cm}$ diameter, and that the electron beam diameter was $\sim 1 \mathrm{~mm}$ at the inner cylinder, in comparison with the $0.1-\mu \mathrm{m}$ scale for target nonuniformity due to Au nucleation.

Count rates were also studied as a function of incident beam current, and found to be linear with current up to a rate of $\sim 8 \times 10^{4} \mathrm{~Hz}$. In practice, beam currents were reduced to ensure maximum count rates during data runs below $10^{4} \mathrm{~Hz}$, and in all cases were kept below $\sim 1 \mathrm{nA}$ to prevent rapid foil breakage. The CDEM discriminators were set to yield maximum signal-to-noise ratios; $A$ was independent of their setting over a broad range. The asymmetry as a function of the spin-rotator coil current was monitored periodically to ensure that $P$ was fully transverse. We discovered, over the course of these measurements, that the value of $P$ was quite stable over the course of days. While it would change from activation to activation, varying between $\sim 0.26$ and 0.32 , once a stable photoemissive surface had been prepared, $P$ would change by less than 0.01 over the course of a week or so of running.

\section{EXTRAPOLATION PROCEDURES}

In Figs. 7 and 8, we present Mott asymmetry data as a function of $t$, the average foil thickness and $\Delta E$, the maximum energy loss which as electron can have suffered and still be detected. Results are shown for the two extremes of incident kinetic energies we investigated: $E=20$ and 100 $\mathrm{keV}$. Uncertainty in the data is dominated by counting statistics, but is occasionally affected by error due to beam

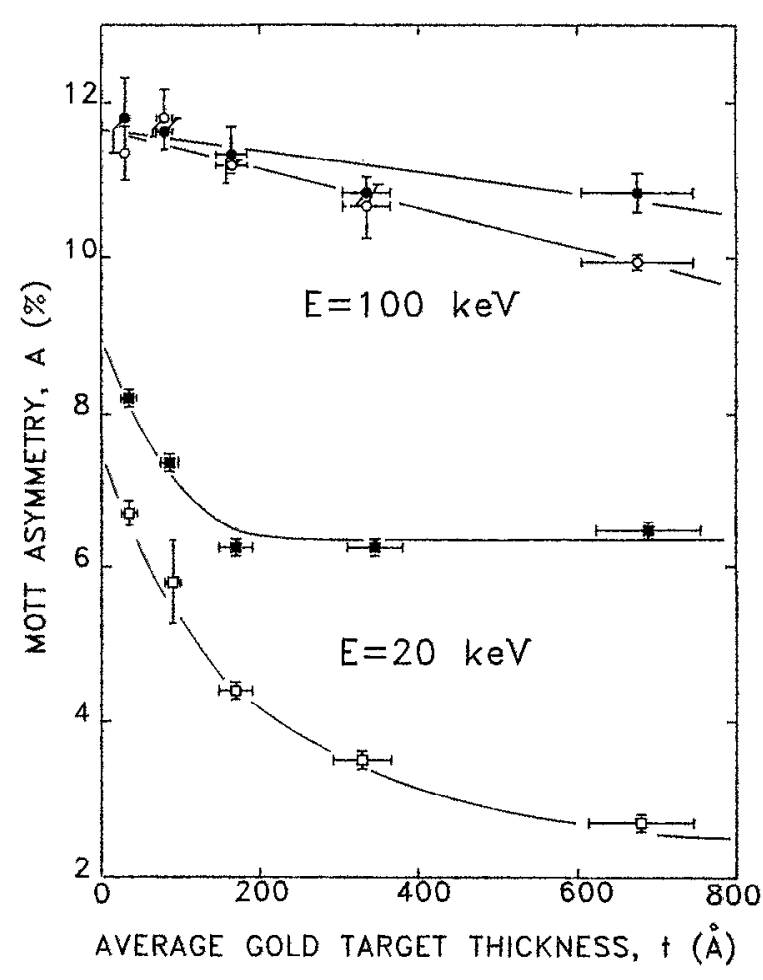

FIG. 7. Mott asymmetry vs target thickness for incident beam energies of 20 and $100 \mathrm{keV}$. The solid data points were taken with $\Delta E=4 \mathrm{eV}$, corresponding to elastic scattering; for the open points, $\Delta E=1300 \mathrm{eV}$. Upper two lines are linear least-square fits to the data. The lower curves are fits to the form $A=a+b e^{-\lambda t}$.

current normalization procedures. Only a few typical error bars are shown in Fig. 8. In both graphs the asymmetry curves, taken on different days with varying electron polarizations, were adjusted relative to each other by the use

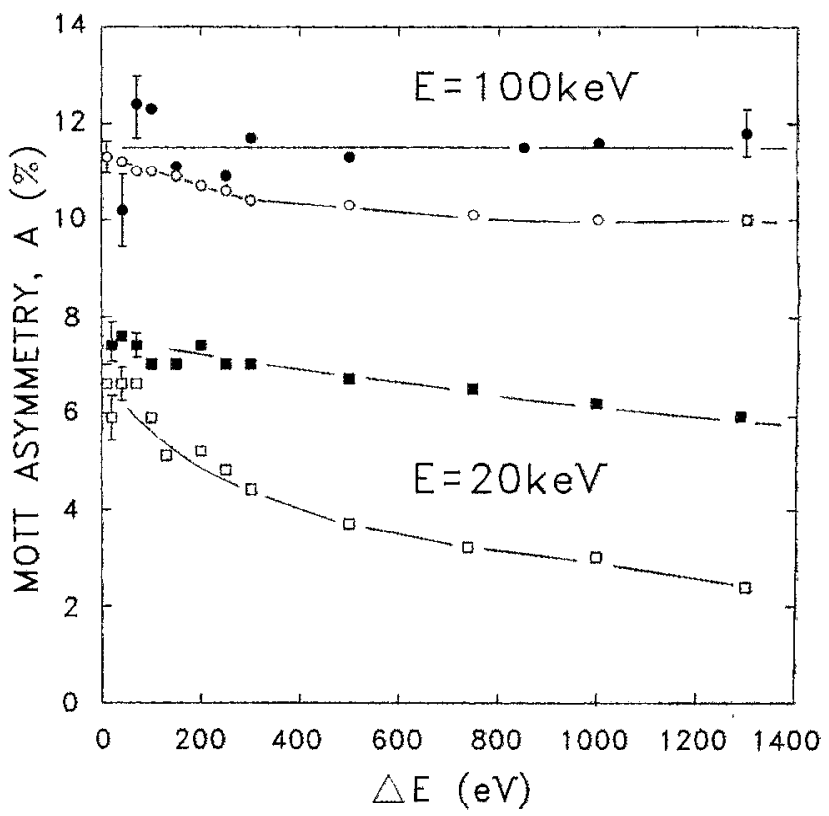

FIG. 8. Mott asymmetry vs maximum energy which electrons can have lost in the target, $\Delta E$. The solid data points were taken with the $34-\AA$ target, the open points with the $682-\AA$ target. Representative error bars are indicated. The solid lines are shown to guide the eye. 


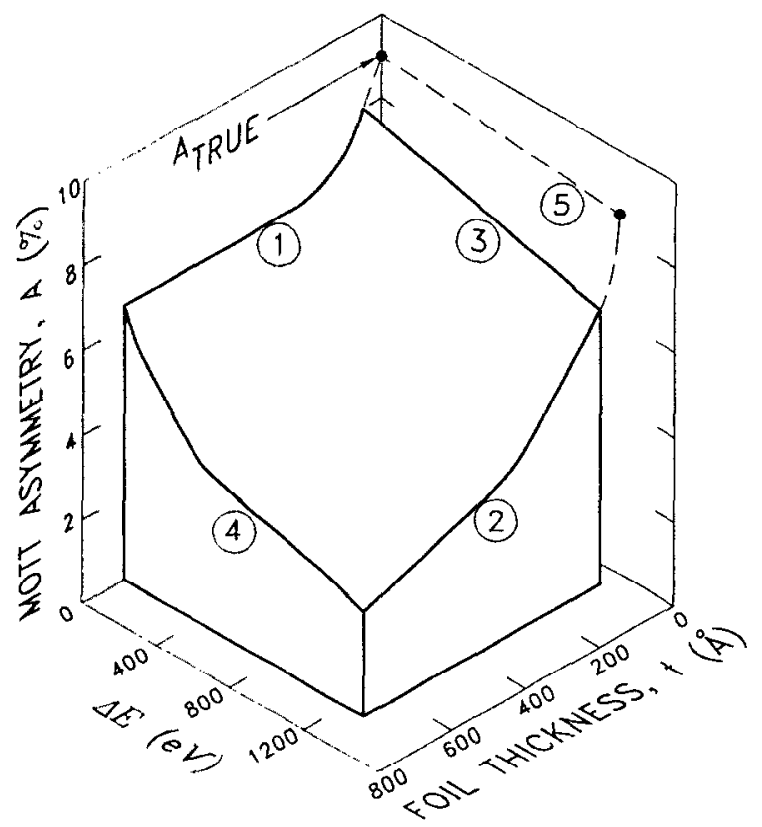

FIG. 9. Schematic representation of $A$ in the $(\Delta E, t)$ plane at $20 \mathrm{keV}$ (see text).

of data sets taken in one run (with constant $P$ ) which contained points common to at least two curves. The error bars shown do not reflect the additional uncertainty caused by this procedure. In order to clarify the following discussion, the $20-\mathrm{keV}$ data of Figs. 7 and 8 are plotted schematically in the three-dimensional $(A, \Delta E, t)$ space in Fig. 9. We will refer extensively to curves $1-5$ in Fig. 9 in a more general sense for all incident energies $E$ : curve 1 is the thickness dependence of $A$ for purely elastic scattering, curve 2 is the thickness dependence with a large (e.g., 1300 eV) $\Delta E$ value, curve 3 is the $\Delta E$ dependence of $A$ for a "very thin" foil, curve 4 represents this dependence for "thick" targets, and curve 5 is the dependence of $A$ on $\Delta E$ for single atoms. Our inability to realize experimentally the $t=0$ limit is reflected in the gap between the $(t=0)$ plane and the data.

Figure 9 clearly shows the consequence of departing from single, elastic scattering conditions (represented by the $A$ axis) in the target. In any Mott extrapolation procedure, failure to obtain " $A$ TRUE" $=$ PS (indicated in the diagram) constitutes a systematic error in the measurement. We now consider $t$ and $\Delta E$ extrapolations in detail, placing particular emphasis on the kinds of systematic errors they can cause. In a general sense, the two extrapolation procedures are roughly equivalent. This is because restriction of $\Delta E$, for example, limits the maximum depth in a target from which an electron can be backscattered and still emerge with enough energy to be detected. Thus a series of diminishing $\Delta E$ measurements corresponds to a series of measurements with targets of decreasing "effective thickness." Conversely, reduction of $t$ limits the typical maximum energy loss in the foil.

\section{A. Potential errors associated with $\Delta E$ extrapolations}

Systematic errors resulting from $\Delta E$ extrapolations can be caused by (1) The use of a target for which the $\Delta E=0$ limit of $A$ is significantly below that of $A_{\text {TRUE }}$. Thus, any dependence of curve 1 on $t$ results in a systematic $\Delta E$ extrapolation error for $A$ with targets of finite thickness. (2) Failure to attain truly elastic scattering conditions when $V_{r}$ equals the GaAs photocathode potential (" $\Delta E$ $=0$ ") or, if $\Delta E$ is not reduced to zero, the use of an inappropriate functional form for $A$ versus $\Delta E$ to extrapolate the data to $\Delta E=0$. We consider problems associated with Sec. IV A 1 to be the most serious, and analyze them first.

\section{Use of thick foils}

The suggestion that high-energy Mott asymmetry measurements employ energy discrimination to approach the $\Delta E=0$ limit and thus reach $A_{\text {TRUE }}$ was first put forth by Boersch et al. ${ }^{31}$ Their proposal was based on the following argument. In the backscattering of polarized electrons from solid targets, it is useful to distinguish (somewhat artificially) between two types of deflections: "plural scattering," in which the electron undergoes several large-angle collisions, and "multiple scattering," corresponding to many small-angle scattering events. To a good approximation, events characterized by a single large $\left(\sim 120^{\circ}\right)$ angle scattering compounded with multiple scattering will result in asymmetries not significantly reduced from $A_{\text {TRUE }}$, while plural backscattering will diminish $A$. Since, on average, the total path length in the target will be larger for plural events than for single, large angle ones, it can be expected that plurally scattered electrons will lose more energy, on average, than those scattered once by $120^{\circ}$. Thus, by discriminating against electrons which have been inelastically scattered, one can expect to observe only single scattering events, with their attendant "true" value of $A$. This argument is strengthened by the fact that the stopping power of high-energy electrons in $\mathrm{Au}$ is appreciable, being of the order of $1 \mathrm{eV} / \AA$ in the energy range under consideration. ${ }^{10}$ Boersch et al. used a conventional Mott double-scattering apparatus with a filter lens of resolution $\sim 1.5 \mathrm{eV}$ to measure $S_{\text {eff }}$ for bulk gold targets between 22 and $48 \mathrm{keV}$. While they were able to obtain values of $S_{\text {eff }}$ closer to the theoretical atomic values of $S$ than any experimental measurements made prior to that time, their results were still lower than theory by about $20 \%$, indicating that, in all likelihood, an experimental systematic problem still existed. ${ }^{31}$

With the advent of concentric-electrode polarimeters of the Farago/Rice design, which allow inelastically scattered electrons to be discriminated against in a straightforward manner, the use of $\Delta E$ extrapolations has become a standard technique for determining $A$. The experimental ease with which they can be carried out compared with thickness extrapolations makes this method particularly attractive. Using arguments similar to that of Boersch et al., several groups have recently proposed that thickness and $\Delta E$ extrapolations should be equivalent, and that suitable calibration of Mott polarimeters can be effected by a $\Delta E$ extrapolation alone. A corollary conclusion of these arguments is the independence of $A$ on $t$ when only elastically scattered electrons are detected (curve 1). 


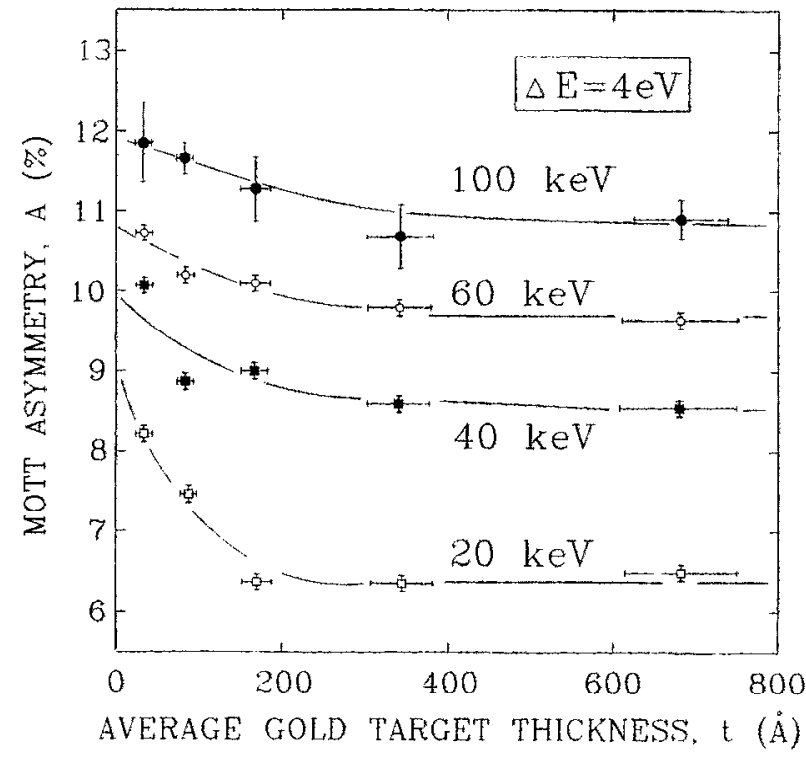

FIG. 10. Mott asymmetry vs average target thickness for elastic scattering. Solid curves represent least-squares fits of the form $A=a+b e^{-\lambda t}$.

Figures 8 and 9 make it immediately clear that the Boersch argument is fallacious, at least for the energy range between 20 and $100 \mathrm{keV}$. The energy dependence of curve 1 in this range is shown in Fig. 10. We note that these data are in qualitative agreement with the only other results available for the target thickness dependence of $A$ with $\Delta E \approx 0$. Dunning et al. ${ }^{39}$ found no dependence of $A$ on $t$ with $10^{3}$ and $2.5 \times 10^{5} \AA$ films for $20 \leqslant E \leqslant 100 \mathrm{keV}$. This can be seen from Fig. 10 to be a consequence of the fact that for $\Delta E \approx 0$, such targets are asymptotically thick. The variation in $A$ above $50 \AA$ at $40 \mathrm{keV}$ has also been seen by the Münster group. ${ }^{7}$

The dependence of curve 1 on $t$ is less surprising when one realizes that the Boersch argument is incorrect for two reasons. The first is the fact that the mean free path for elastic scattering is about a factor of five shorter in gold between 10 and $100 \mathrm{keV}$ than is that for inelastic scattering. ${ }^{40}$ Thus, electrons can be expected to undergo several elastic collisions, on average, before they inelastically scatter. Moreover, the angular dependence of the inelastic scattering cross section is strongly peaked in the forward direction, whereas the elastic cross section falls off much more slowly in angle. ${ }^{40,41}$ As a result, electrons which have been plurally scattered to $120^{\circ}$ have a higher probability of being elastic than do electrons that have been multiply scattered through only a few degrees. The importance of plural elastic scattering in reducing $A$ is apparent in Fig. 10; that it contributes to the total number of elastic scattering events is suggested by the 60 - and $100-\mathrm{keV}$ data of Fig. 11(a), where $N$ appears to increase at least quadratically with $t$ before the target depth becomes so great that some energy loss is inevitable, and the asymptotic thickness regime is reached. Such increases are not apparent in the 20- and 40-keV data, perhaps because there are only two data points in the region where $N$ rises at these energies. When $N$ is taken under quasielastic scattering conditions $[\Delta E=100,1300 \mathrm{eV}$; Figs. 11(b), 11(c) $]$ a "growth region" with a positive second derivative is apparent.

The data of Fig. 11 (a) point out the second problem with the Boersch argument: the implicit assumption that the electrons lose energy continuously in the target. In fact, the electrons lose energy in discrete quantities on a colli- (a) $\Delta E=4 e V$

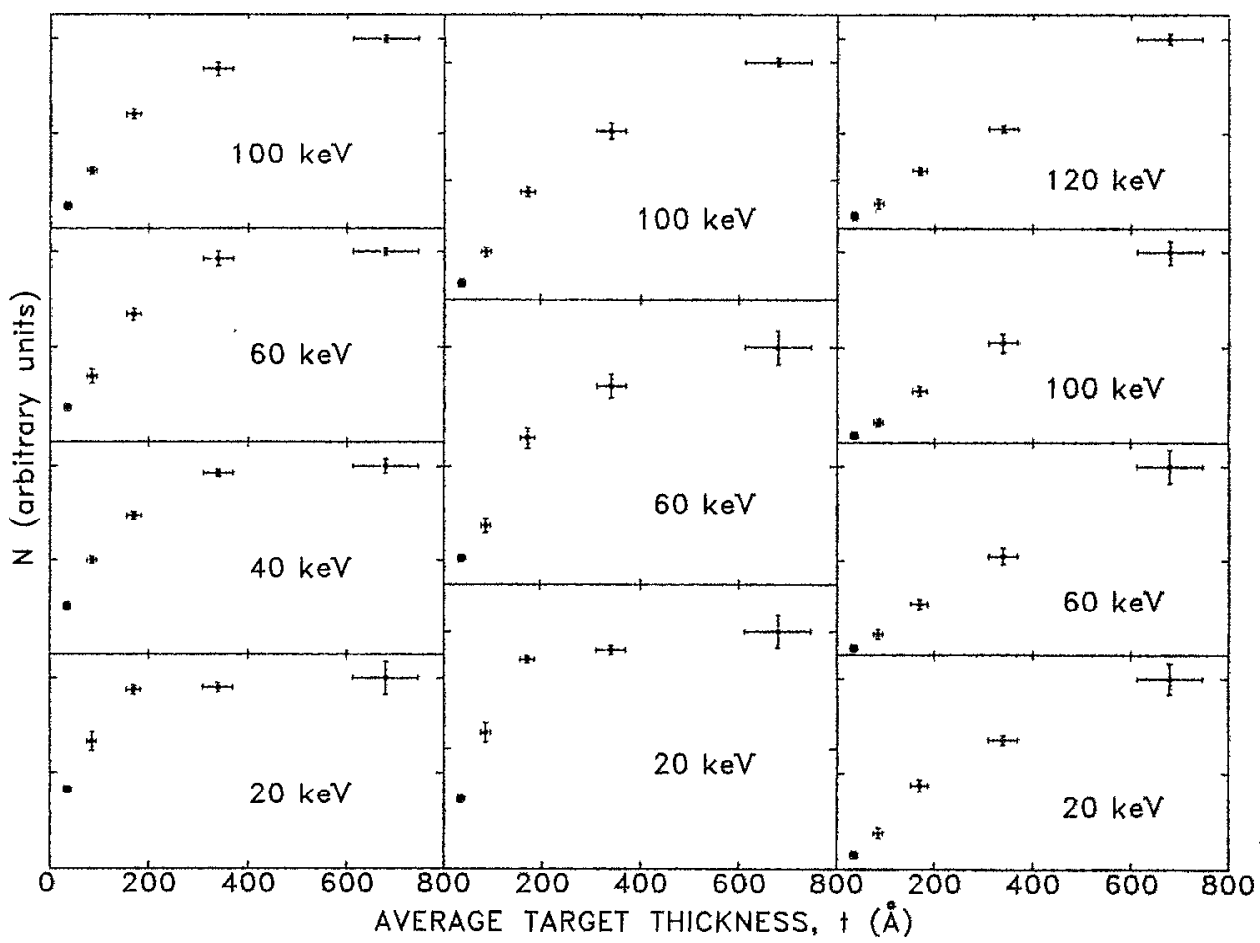

FIG. 11. Total scattered electron counts vs target thickness for various values of $E$ and $\triangle E$. The uncertainties in $N$ are dominated by beam current normalization errors; thickness uncertainties are relative and are those required to yield a reduced $\chi^{2}$ of unity for a linear fit to the $E=120 \mathrm{keV}, \Delta E=1300 \mathrm{eV}$ data (see Sec. IV B). 


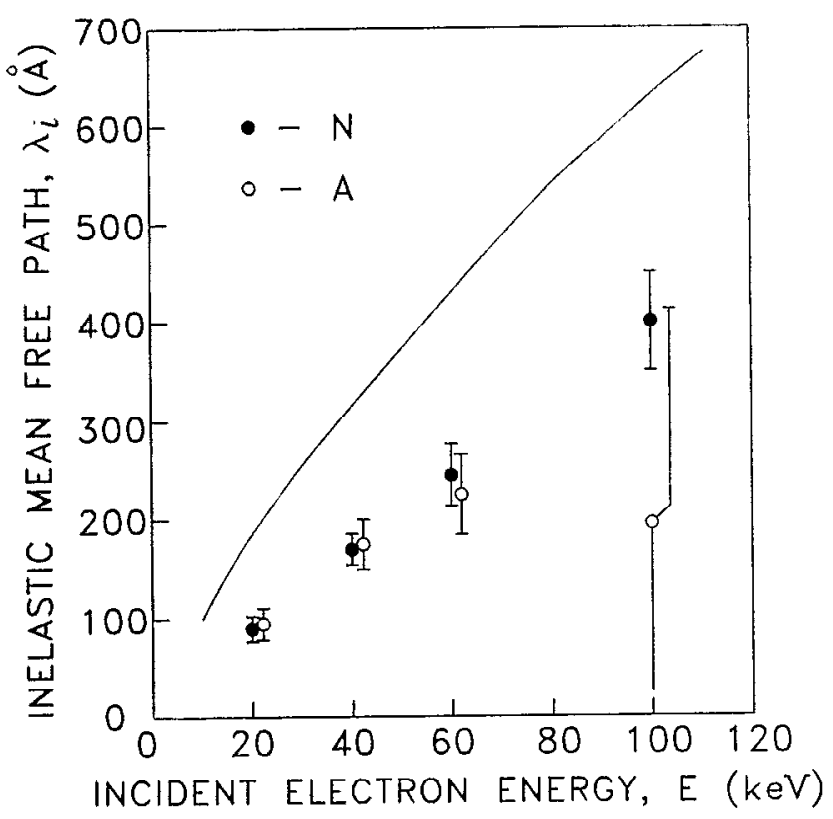

FIG. 12. Values of the inelastic mean free path $\lambda_{i}$ for electrons in $\mathrm{Au}$ as a function of their energy, as obtained from exponential fits to $A$ and $N$ vs $t$ data (see text). Solid line represents the theoretical data of Ref. 40.

sion-by-collision basis, ${ }^{42}$ and the probability of energy loss can be characterized by an inelastic mean free path, equal to average energy lost per collision, divided by the stopping power. ${ }^{10,15}$ The average stopping power as calculated in the Bethe theory is, indeed, above $1 \mathrm{eV} / \AA$ at these energies, but if the electrons lost energy continuously at this rate, the asymptotic region of Fig. 11 (a) would begin at about $10 \AA$, instead of $300 \AA$. Thus, a significant fraction of the backscattered electrons can travel several inelastic mean-freepath lengths before losing any energy. The contention that plurally scattered electrons must travel further in the foil than ones singly scattered to $120^{\circ}$, while true, in no way rules out the importance of plural scattering events in an elastic experiment.

We have extracted from our $\Delta E=4 \mathrm{eV}$ data a rough estimate of the inelastic mean free path in gold for 20 $\mathrm{keV}<E<100 \mathrm{keV}$. By calculating weighted least-squares fits of $A$ vs $t$ to the phenomenological form $a+b e^{-\lambda t}$, and of $N$ vs $t$ to $d\left(1-e^{-\lambda t}\right)$, and by taking a crude estimate of the average path length in a target of thickness $t$ to be $3 t / 2$ for $120^{\circ}$ electron detection, we have obtained from fit values of the parameter $\lambda$ estimates for $\lambda_{i}(=3 / 2 \lambda)$, the inelastic mean free path. These are shown in Fig. 12, where they are compared with the theoretical calculations of Misell. ${ }^{40}$ It should be pointed out that our estimates represent a lower limit to the true value of $\lambda_{i}$, because plural scattering causes the average path length in the target to be somewhat greater than $3 t / 2$.

Systematic errors associated with plural elastic scattering can be reduced or eliminated by the use of foils so thin that the probability of plural scattering becomes negligible, and/or by making measurements at high energy, where the dependence of curve 1 on $t$ is less pronounced (Fig. 10). With regard to target thickness, the question naturally arises: "How thin is thin enough?" Campbell et al., in their discussion of the Boersch argument, ${ }^{10}$ point out that the elastic scattering cross section is bigger than the inelastic one, and raise this issue, correctly suggesting that the deviation of the Boersch et al. results from theory is due, at least in part, to the use of bulk gold targets. Unfortunately, due to an error in their data analysis procedure (see Sec. IV $\mathrm{B} 2$ and the Appendix), they conclude that $300-\AA$ targets are thin enough to eliminate the effects of plural scattering over the range from 20 to $100 \mathrm{keV}$. Our data (Fig. 10) suggest that even $34-\AA$ foils are inadequate to completely eliminate systematic errors of this type, although at $100 \mathrm{keV}$, these errors are small. By $300-\AA$, the asymptotic thickness region has essentially been reached at all energies. Thus, a calibration of our apparatus based on a $\Delta E$ extrapolation at, e.g., $20 \mathrm{keV}$ with a "thin" foil $200-\AA ̊$ thick would be in error by almost $30 \%$ ! At $100 \mathrm{keV}$, the error would be less than $10 \%$. A similar situation is illustrated in Fig. 5 of Uhrig et al., ${ }^{7}$ where an extrapolation is made to a value of $A$ only slightly higher than its asymptotic value, when the true intercept is certainly above 0.11 . Unfortunately, when asymmetry measurements are made with targets $<50$ - $\AA$ thick, statistical uncertainties and large Formvar background subtractions can severely limit accuracy, especially at high energy. Thus for high precision, calibrations are best done using thickness extrapolations (see Sec. IV B below).

\section{Extrapolation and energy resolution errors}

Fven if measurements are made at a high enough energy that curve 1 is effectively flat within acceptable error limits for $P$, systematic error can still result from conditions listed under (2) above. If $A$ is not measured directly for elastic scattering, but is instead extrapolated to $\Delta E=0$ from measurements made at lower values of $V_{r}$, the functional form of $A$ vs $V_{r}$ should, in principle, be known to allow for proper fitting and extrapolation of the data. Unfortunately, such functions depend not only on the physics of electron energy loss in the foil, but also on the type of apparatus, varying, for example, with the details of the electron-optical elements and detection efficiency of the analyzer. ${ }^{3,10,32,33,35,38}$ We note, in this context, and in contrast to several statements which can be found in the literature, that linear behavior of $N$ vs $\Delta E$ does not guarantee linearity of $A$ vs $\Delta E$ (see, e.g., Figs. 3 and 4 of Ref. 12). Similarly, nonlincarity in $N$ vs $\Delta E$ may not cause nonlinearity in the corresponding value $A$ vs $\Delta E$. Curve 3 of Fig. 9, for example, was linear at all energies we investigated, while corresponding $N$ vs $\Delta E$ plots, while monotonic, were nonlinear, with several inflection points over their $\Delta E$ range. If the retarding potential $V_{r}$ is defined by the entrance of the electron multiplier, ${ }^{10,14}$ electron detection efficiency can depend strongly on $\epsilon$, the individual electron energy loss, and great care must be exercised in extrapolating $A$ to the $\Delta E=0$ limit. This problem is discussed in Sec. IV $B 2$ and in the Appendix.

In practice, issues regarding the functional form of $A$ vs $\Delta E$ can be disregarded if only purely elastic electrons are detected, or if measurements are made over a sufficiently 
narrow range of $\Delta E$ values that $A$ depends only to first order on $\Delta E$, i.c., any physically reasonable functional fitting form gives the same intercept (see, e.g., the discussion in Ref. 14). With thin foils, the maximum value of $\Delta E$ can be of the order of $\mathrm{keV}$ before this condition is not met. At $E=20 \mathrm{keV}$ with our $682-\AA$ foil however, $\Delta E$ should be limited to $200 \mathrm{eV}$.

A second source of $\Delta E$ extrapolation error which is more difficult to eliminate is caused by electron energy analyzers with poor energy resolution. If curves 3 or 4 are measured using analyzers with energy resolution $W$, they are shifted to the left of the "true" curves (which would be obtained using analyzers with perfect energy resolution) by an amount roughly equal to $W / 2$. Some of our early RFA designs had $W$ values as large as $100 \mathrm{eV}$. While measurements of curve 3 would thus have been affected little by the use of these analyzers, curve 4 at $20 \mathrm{keV}$ (Fig. 8 ) would have had an intercept of $\sim 6.2 \%$ instead of $6.8 \%$, or a $10 \%$ error. Systematic error due to large $W$ 's can be viewed alternatively as a failure to extrapolate the $A$ vs $\Delta E$ data to a value of $\Delta E=-W / 2$, where the real $\Delta E=0$ limit is found. Experimentally, this can be done properly by raising $V_{r}$ until only the very "tail" of the retarding-field curve (Fig. 5) remains, yielding an arbitrarily good energy resolution. This problem is discussed in more detail in the Appendix.

In summary, extrapolation errors in curves 3 or 4 can be eliminated by restricting the range over which $A$ vs $\Delta E$ data are taken, and by careful reduction of $W$, or extrapolation of $A$ to the real $\Delta E$ limit, which may correspond to values of $V_{r}$ above the source photocathode potential. Again, if statistical uncertainty is not an issue, these problems can be minimized at the outset by running with thin targets at high $E$.

\section{B. Potential errors associated with target thickness extrapolations}

Systematic errors associated with target thickness extrapolations can be caused by the following.

\section{Large energy-acceptance width of the detectors}

Consider curve $5, A$ vs $\Delta E$ with a single atom target. Inelastic processes will cause $A$ to deviate, at some level, from the pure, elastic, single atom case, resulting in the slope of this curve. Thus, a finite value of $W$ (which may be as large as $10 \mathrm{keV}$ in the case of surface barrier detectors used in "standard" Mott polarimeters ${ }^{15}$ ) will cause an error in the measured value of $A$. In practice, we expect this problem to be negligible, except for the lowest incident energies when surface barrier detectors are used. As mentioned earlier, the elastic angular-differential cross section for scattering to $120^{\circ}$ is much larger than is that for inelastic scattering. ${ }^{41}$ Moreover, the differential inelastic cross section at a given (large) angle falls off roughly as the second power of energy loss, meaning that the great majority of inelastic collisions at these incident energies are associated with energy losses of less than $500 \mathrm{eV} .^{15,43} \mathrm{Such}$ collisions typically involve ionization of valence shell electrons and would cause $A$ to differ negligibly from PS. Thus, curve 5 can be expected to be virtually independent of $\Delta E$ until $\Delta E$ is comparable to $E$. With Farago/Rice polarimeters, this condition is easily precluded experimentally. Even with our $\sim$ ten-monolayer thick $34-\AA$ target, $A$ is independent of $\Delta E$ at $100 \mathrm{keV}$; curve 3's slope at this energy is $+1.2(3.7) \times 10^{-6} \mathrm{eV}^{-1}$. At $20 \mathrm{keV}$, curve 3 does slope downward, a change attributable to the increasing importance of elastic plural scattering (which is very small at all $\Delta E$ on curve 3 at $100 \mathrm{keV}$ ) compounded with smallangle inelastic scattering as $\Delta E$ is increased. If $t$ could be decreased further from $34 \AA$, however, we would expect to see the trend evident in the bottom two curves of Fig. 8 $(t=682 \rightarrow 34 \AA)$ continue, with a limiting curve essentially independent of $\Delta E$ over the range of the data. We thus conclude that systematic errors due to finite detector acceptance energy can be disregarded in all practical cases.

\section{Uncertain fitting form for $A$ vs $t$}

A much more serious problem is that of target thickness extrapolation. To be free of systematic error, the extrapolation must yield a $t=0$ intercept corresponding to curve 5 , which we will consider, for this discussion, to have a constant value equal to $A_{\text {TRUE. }}$. Thickness extrapolations have, in principle, an advantage over $\Delta E$ extrapolations, in that they can be made using a functional form which is much less sensitive to the details of the apparatus. While target morphology can, at some level, affect the fitting form, electron-optical and detection efficiency effects are eliminated. Unfortunately, there exists no standard, generally accepted model for the form of $A$ vs $t$ as a function of $E$ and $\Delta E .^{15}$ A number of theories have been proposed, ${ }^{44,45}$ but none are unambiguously applicable to the experiments which have been done. Generally, researchers in the field have chosen a single functional form with which they extrapolate their data, and have ignored other possible fitting forms. This is an acceptable procedure only if the foils used in the experiment are so thin that the dependence of $A$ on $t$ is of first order in $t$. If thicker foils are used and secondorder terms are appreciable, then different fitting forms (none of which can be rejected solely on the basis of theoretical considerations) may yield statistically different values for the $t=0$ intercept. ${ }^{15}$ Usc of one form exclusively may thus result in a systematic error in $A_{\text {TRUE }}$. Fletcher et $a l^{15}$ have suggested that, in the absence of better theoretical guidance, the appropriate course of action under these circumstances is to quote an average value of all the intercepts, while increasing the quoted uncertainty to include all of the intercepts at the $2 \sigma$ level.

To reduce the systematic uncertainty associated with this procedure (which Fletcher et al. estimate in their experiments to be about $3 \%$ ), i.e., to remove the ambiguity associated with our lack of knowledge of the "correct" fitting form, two obvious steps are to reduce the range of $t$ and/or $\Delta E$ until the $t=0$ intercept obtained from the extrapolation procedure is independent of the fitting form used. [Lowering of $\Delta E$ reduces the effective thickness from which electrons can be scattered and still be detected. The effect of reducing $\Delta E$ is clearly illustrated both in our Fig. 7 and Fig. 5 of Ref. 7. The use of Farago/Rice polarimeters 
is particularly useful in this context; measurements made with "standard" Mott polarimeters having $W \sim 10 \mathrm{keV}$ exhibit $A$ vs $t$ slopes ("depolarization constants") several times larger than those obtained with concentric-electrode devices. ${ }^{4,5,10,11,15,45}$ ] The lower two curves of Fig. 7 illustrate the difficulty of doing this at low energy. Even with $\Delta E=4 \mathrm{eV}$ and the thinnest foils used in Mott polarimetry to date, $A$ vs $t$ is grossly nonlinear. In that both curves must converge at $t=0$, it is clear that the linear $t$ region for both values of $\Delta E$ begins well below $34 \AA$. In contrast, at $100 \mathrm{keV}, A$ is essentially linear with $t$ over the entire foil thickness range we investigated.

a. Functional forms and fitting procedure. Given the difficulty of making precise asymmetry measurements with foils less than $50-\AA$ thick, and the nonlinearity of curves 1 and 2 even below $34 \AA$ when $E=20 \mathrm{keV}$, it is clear that precise thickness extrapolations for the purpose of instrument calibration must be made at high energy. Even then, nonlinearities can cause some degree of error. In order to illustrate some of these ideas, we have fit the data of Fig. 10 and the $20-\mathrm{keV}$ data of Fig. 7 to a variety of functional forms:

(a) $A=a+b t$. This is the simplest equation to use, and although there is no theoretical justification for it (save the fact that it must be correct for sufficiently thin targets) it has been considered exclusively by the majority of investigators.

(b) $1 / A=a+b t$. This form was first proposed by Wegner $^{44}$ as a result of his extensive analysis of plural scattering effects and has been used in several studies. Greenburg et al. consider its applicability in detail. ${ }^{46}$

(c) $A=a+b N$. This form, first proposed explicitly by Fletcher et al., ${ }^{15}$ has the advantage that it implicitly includes higher-order scattering effects.

(d) $1 / A=a+b N$. A variation of (b) and (c) above.

(e) $A=a+b e^{-\lambda t}$. Phenomenologically, this form appears to describe the data well and, to the extent that $N$ is proportional to $\left(1-e^{-\lambda t}\right)$, it is equivalent to form (c) above.

Functions (c), (d), and (e), in addition to their implicit inclusion of triple- and higher order scattering, also have an advantage over the $t$-dependent functions in that they incorporate the necessary asymptotic independence of $A$ on $t$ automatically. A number of authors have used forms such as $A=e^{-\lambda t}, A^{-1 / 2}=a+b t$, and [see Eq. (5)] $\Delta=a+b t$, based solely on the goodness of fit they obtained with these forms. We considered the latter two forms, and found them to give intercept results in between those of (a) and (b) above; the exponential function is of course just a special case of (e), but requires an asymptotic value of $A=0$ in all cases, which is not physical.

The linear fits to equations (a)-(d) were done using the procedure described in Ref. 47; the exponential fits were made with a standard nonlinear least-squares also described in this reference. Initial fits were obtained with weighting according to the uncertainties in $A$ alone. Using these fits, an additional uncertainty was added in quadrature to $A$ (or $1 / A$ ) equal to the fit slope at the abscissa of the given datum times the uncertainty either in $N$ or $t$. This procedure converged after one iteration for all fits except those with reduced $\chi^{2}$ values greater than 10 . While the absolute values of $t$ were estimated from $\mathrm{x}$-ray fluorescence measurements, their relative values [which are the only critical ones for determining $A(t=0)$ ] were taken from the relative lengths of gold wire evaporated to produce them. Although the individual targets were made under identical conditions, this does not guarantee that final target weight is proportional to the length of the bulk gold because, e.g., the gold atom sticking coefficient might vary with the amount of gold already on the surface. Thus, to check that the target average areal densities were proportional to evaporated gold weight, we "weighed" the foils by measuring $N$ versus the length of wire used to produce the target yielding that $N$ at the highest practical values $E$ and $\Delta E$, where the relationship between $N$ and the actual $t$ should be most linear (see Fig. 11). These data [which are essentially those at the top of Fig. 11(c) multiplied by a scale factor on the $t$ axis] exhibit small deviations from linearity which are almost certainly due to the nonlinear nature of the scattering process at these energies, and not a lack of proportionality between $\mathrm{Au}$ wire length and $t$. Nonetheless, for the sake of conservatism, we quote $t$ uncertainties which correspond to a reduced $\chi^{2}$ of unity for a linear fit to these data. Thus, the relative uncertainty of the four largest average thicknesses is $10 \%$. The thinnest target's thickness is uncertain by $14 \%$ due to additional uncertainty in the wire length measurement procedure.

Uncertainties in $N$ were determined by adding in quadrature the statistical uncertainties in the raw accumulated counts and the uncertainty due to the beam current normalization procedure. This latter contribution dominates the $N$ error bars in almost all cases. Occasionally, $N$ for a given target would vary by an amount larger than that suggested by the uncertainties for one run. This was attributed to pinholes in the targets, which could be observed in some films with an optical microscope. Such variations did not affect the observed asymmetries, and occurred in only one data set we fitted: $E=100 \mathrm{keV}, \Delta E$ $=100 \mathrm{eV}$ (see below). In this case, both values of $N$ were used in the fit, but elimination of the lower value does not affect the intercept.

The $t=0$ intercepts and reduced $\chi^{2}$ values for the various fits are given in Table I. (Note that the data are not normalized to constant electron polarization, and that the intercept values vary with energy because of the variation of $S$ with $E$.) A number of points are apparent. Below $E=100 \mathrm{keV}$, the data are not linear in $t$ (this is trivially obvious from the figures), as evidenced by the large reduced $\chi^{2}$ values. The $N$-dependent and exponential fits are roughly linear for all $E$ and $\Delta E$ values, with a notable exception at $40 \mathrm{keV}$, where the poor $\chi^{2}$ values can be attributed to the rather large nonmonotonic variation of $A$ for the three thinnest targets. The cause of these nonstatistical variations is not understood; no obvious problems are apparent in the raw data and these targets produced "reasonable" results in subsequent runs at other $E$ and $\Delta E$ values. We thus cannot justify eliminating the $40-\mathrm{keV}$ results. At low energies, the intercepts of the linear $t$ forms 


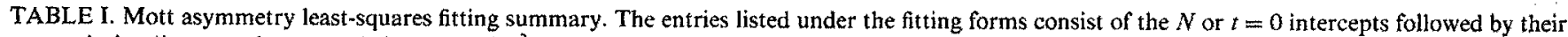

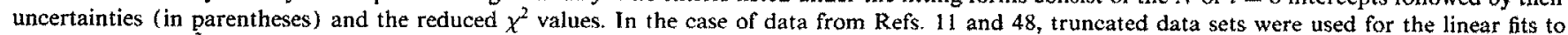

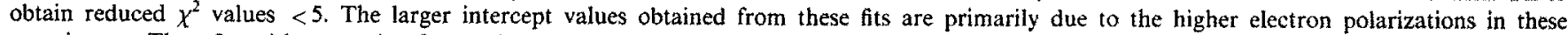
experiments. The $t$ fits with correction for partial film coverage are also listed (see text).

\begin{tabular}{|c|c|c|c|c|c|c|c|c|c|}
\hline$E(\mathrm{keV})$ & $\Delta E(\mathrm{eV})$ & $A$ vs $t$ & $\begin{array}{c}A \text { vs } t^{\prime} \\
\text { Coverage } \\
\text { corrected }\end{array}$ & $1 / A$ vs $t$ & $\begin{array}{l}1 / A \text { vs } t \\
\text { Coverage } \\
\text { corrected }\end{array}$ & $A$ vs $N$ & $1 / A$ vs $N$ & Exponential & $\begin{array}{c}\text { Exponential } \\
\text { Coverage } \\
\text { corrected }\end{array}$ \\
\hline \multirow[t]{2}{*}{20} & 1300 & $5.44(8)$ & $5.86(10)$ & $6.44(14)$ & $7.22(32)$ & $5.97(8)$ & $6.73(13)$ & $6.64(10)$ & $7.57(31)$ \\
\hline & & 47 & 32 & 4.5 & 2.3 & 11 & 2.0 & 0.5 & 0.2 \\
\hline \multirow[t]{2}{*}{20} & 4 & $7.72(5)$ & $7.90(6)$ & $7.88(5)$ & $8.11(7)$ & $9.69(15)$ & $10.66(28)$ & $9.73(39)$ & $10.36(68)$ \\
\hline & & 62 & 53 & 52 & 43 & 0.5 & 0.8 & 6.1 & 11 \\
\hline \multirow[t]{2}{*}{40} & 4 & $9.93(4)$ & $10.06(5)$ & $10.07(4)$ & $10.22(5)$ & $10.64(8)$ & $10.85(7)$ & $10.31(13)$ & $12.16(69)$ \\
\hline & & 86 & 75 & 77 & 64 & 45 & 39 & 15 & 12 \\
\hline \multirow[t]{2}{*}{60} & 4 & $10.85(5)$ & $10.94(6)$ & $10.89(5)$ & $10.98(6)$ & $11.22(8)$ & $11.26(8)$ & $11.30(7)$ & $11,64(18)$ \\
\hline & & 11 & 10 & 11 & 9.0 & 4.1 & 4.1 & 2.2 & 1.6 \\
\hline \multirow[t]{2}{*}{100} & 4 & $11.65(23)$ & $11.72(25)$ & $11.68(24)$ & $11.75(26)$ & $11.96(31)$ & $11.99(33)$ & $12.30(54)$ & $12.76(1.23)$ \\
\hline & & 0.8 & 0.8 & 0.7 & 0.7 & 0.2 & 0.2 & 0.3 & 0.3 \\
\hline \multirow[t]{2}{*}{100} & 100 & $11.86(9)$ & $11.93(9)$ & $11.88(9)$ & $11.94(10)$ & $11.92(9)$ & $11.94(10)$ & $12.00(13)$ & $12.11(21)$ \\
\hline & & 0.7 & 0.7 & 0.6 & 0.6 & 0.9 & 0.9 & 0.7 & 0.7 \\
\hline \multirow[t]{2}{*}{120} & 1300 & $17.40(11)$ & $\cdots$ & $17.55(12)$ & $\cdots$ & $\cdots$ & $\cdots$ & $17.69(12)$ & $\cdots$ \\
\hline & 11) & $\begin{array}{c}2.3 \\
(t<1100 \AA)\end{array}$ & $\cdots$ & $\begin{array}{c}2.4 \\
(t<1100 \AA)\end{array}$ & $\cdots$ & $\cdots$ & $\cdots$ & $\begin{array}{c}2.7 \\
\text { (all data) }\end{array}$ & $\cdots$ \\
\hline \multirow[t]{2}{*}{616} & $\sim 6 \times 10^{4}$ & $33.67(19)$ & $\cdots$ & $34.38(24)$ & $\cdots$ & $\cdots$ & $\cdots$ & $33.72(20)$ & $\cdots$ \\
\hline & 48) & $\begin{array}{c}0.6 \\
(t<8000 \AA)\end{array}$ & $\cdots$ & $\begin{array}{c}1.0 \\
(t<8000 \AA)\end{array}$ & $\cdots$ & $\cdots$ & $\cdots$ & $\begin{array}{c}3.6 \\
\text { (all data) }\end{array}$ & $\cdots$ \\
\hline
\end{tabular}

are significantly different from those of the exponential and $N$ formulae, which tend to agree with each other. This is not surprising, given the approximate equivalence of (c) and (e). At $100 \mathrm{keV}$ all of the fitting forms give intercepts which are essentially identical statistically. This means that we can view elastic scattering at $100 \mathrm{keV}$, within the statistical uncertainty associated with this measurement $(\sim 2.5 \%)$, to be first order in $t$. Thus, unlike the situation considered by Fletcher et al. ${ }^{15}(E=94 \mathrm{keV}, \Delta E \sim 10 \mathrm{keV})$, the accuracy of $A$ determined from this measurement is limited not by uncertainty in the fitting form, but by statistics alone. In this context, we note that the exponential fits suffer one disadvantage compared with linear fits: as $E$ is increased and the data become more linear in $t$ or $N$, uncertainty in the three fitting parameters tends to increase, removing to some extent the benefits associated with improved clustering of the intercepts about their average. The larger uncertainties in the intercepts for the exponential fits at all energies is a general symptom of this problem.

Making measurements at high energy does not, in and of itself, guarantee that variations in $A$ will be linearly dependent on $t$. We also display in Table I the results of fits to the data of Refs. 11 and 48 . Hodge et al. ${ }^{11}$ report asymmetries at $E=120 \mathrm{keV}$ and $\Delta E=1300 \mathrm{eV}$ for target thickness $\lesssim 1500 \AA$. Their data in its entirety are clearly described best by the exponential form, although $\chi^{2}$ values $<3$ may be obtained with a linear $t$ fit if the data point at $\sim 1500 \AA$ is eliminated. Thus, these asymmetries are only marginally linear in $t$, a consequence of the relatively large values of $\Delta E$ and target thickness used in this experiment. Even the intercepts of the Brosi et al. data, ${ }^{48}$ taken at 616 $\mathrm{keV}$, exhibit nonstatistical scatter at the $2 \%$ level, again because of large $t$ and $\Delta E$ values. An exponential fit describes the variation of $A$ with $t$ over the broadest thickness range with these data as well.

b. The effect of target morphology. When target foils have varying morphology, the parameterization and fitting of $A$ in terms of the simple average target thickness $t$ will, at some level, lead to systcmatic error in the $t=0$ intercept. (Interestingly, this issue had not been discussed at all in the literature prior to last year, ${ }^{5}$ although many investigations have used foils of sufficient thinness that their coverage was almost certainly nonuniform.) A question naturally arises as to whether the differences between the intercepts for $t$ and $N$ fits is due to morphology effects alone. We have attempted to understand qualitatively the effect that target nonuniformity due to nucleation has on the extrapolated asymmetrics obtained from the $t$-dependent fits. Our foils are characterized by two known parameters, average areal density, $\sigma(t=\sigma / \rho$, where $\rho$ is taken as the bulk volume density of gold), and $p$, the percent coverage of the target film. A first-order correction, which is all that is really justifiable, given our poor knowledge of nucleated target morphology, can be made for nonuniformity by assuming that reduction of $A$ is caused exclusively by double large-angle scattering. ${ }^{44,46}$ The amount of reduction should thus be roughly proportional to the average path length in the target for single $120^{\circ}$ scattering. This equals $3 t / 2$ for a uniform film but increases when voids are present, because of the higher average thickness of the nucleated regions. By using the simple model that the target may be partitioned into a single layer of contiguous cubic volumes, of which a percentage $p$ are filled with $\mathrm{Au}$, and whose heights $h$ are such that the average thickness of gold in all the cubes is $t(h=\sigma / p \rho)$, the average path length in the gold, including alterations due to exil and entrance through the vertical sides of gold cubes, can be calculated. 


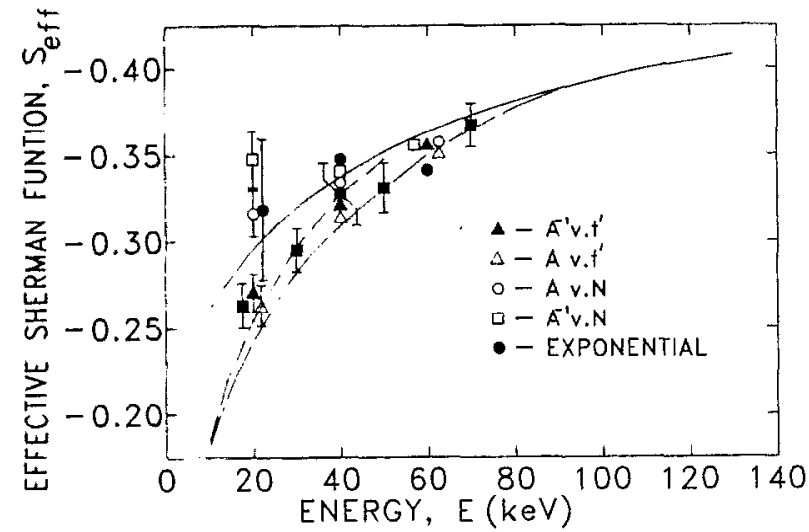

FIG. 13. Effective Sherman function vs incident electron energy, with some representative uncertainties indicated. The solid line represents the theoretical calculations of Ref. 51; the absolute measurements of Ref. 7 are indicated by the short dashed line, and the substantially equivalent results of Refs. 12 and 14 are indicated by the broken line. The solid squares are the modified data of Campbell et al. (Ref. 10; see text), normalized at $100 \mathrm{keV}$ to the theory. Our results, also normalized at 100 $\mathrm{keV}$, are plotted for different extrapolation forms. Error bars indicated at $20 \mathrm{keV}$ include both statistical and normalization uncertainties.

The value of $p$ for a given target was taken from a fit of all the coverage data (see Sec. II) to $p=a \log t+b$, a form suggested by previous coverage results for $\mathrm{Au}$ on carbon films. ${ }^{49}$ We thus calculated "effective average thicknesses" $t^{\prime}$ for the targets, corresponding to the thicknesses uniform films would have which give the same average path length for single $120^{\circ}$ scattering which our actual nucleated targets give. These were $t^{\prime}=71 \AA(34 \AA), 125 \AA(85 \AA), 211$ $\AA(171 \AA), 369 \AA(341 \AA)$, and $682 \AA(682 \AA)$, where the actual average target thicknesses are given in parentheses.

While the corrections to $t$ for the thinnest targets are large, the changes in the fit values do not account for the differences between the various $t$ and $N$ fits, ${ }^{50}$ except for the $E=100, \Delta E=100 \mathrm{eV}$ data, which are statistically equivalent in any case (see part $d$ below).

c. Results; energy dependence of $A_{\mathrm{TRUE}}$. These results suggest that the appropriate parameter to use when extrapolating asymmetry data taken with different target thicknesses is the total scattered intensity $N$. The exponential form (e) appears to give reasonable results as well. The $N$ fits have the advantage that careful measurements of $t$ are not required, and target morphology is irrelevant.

Ultimately, the best test of whether a fitting function is proper is if the values of $A_{\text {TRUE }}$ it yields for a given $P$ have the same energy dependence as do the theoretical results for $S .{ }^{10}$ This type of comparison has been made in several previous experiments between 10 and $115 \mathrm{keV}$ which involved $\Delta E$ rather than thickness extrapolations. ${ }^{7,10,12,14}$ In these experiments, whose results are shown in Fig. 13, either electrons of known polarization were used to determine $S_{\text {eff }}$ absolutely, ${ }^{7,10}$ or the data were taken with constant (unknown) $P$ and normalized to $A$ at $100 \mathrm{keV}$, where $S_{\text {eff }}$ was assumed to equal $S .{ }^{12,14}$ In all cases, the data fall below the theoretical Sherman function below $\sim 60 \mathrm{keV}$. As a result of the discussion of Sec. IV A 1 above and the data of Fig. 10, this deviation is simply understood in terms of the increasing effect of plural elastic scattering in the targets used in these experiments. The data of Uhrig et $a l^{7}$ exhibit a less severe departure from theory because of the relatively thin target $(100 \AA)$ employed in their experiment. The values of $S_{\text {eff }}$ attributed to Campbell et al. ${ }^{10}$ in Fig. 13 have been modified from the values they report. Their published data do follow the theoretical energy dependence of $S$, which they attribute to the combined use of thin foils and $\Delta E$ extrapolation. While this idea is physically correct, as we have demonstrated, their target thickness $(300 \AA)$ is well into the asymptotic region of curve 1 at $20 \mathrm{keV}$. The fact that their reported asymmetries do not deviate from theory at low energy is an artifact of the improper extrapolation of their data to $\Delta E=0$. This problem is rectified in the Appendix.

By using target thickness extrapolations in combination with zero energy-loss measurements, the problem of plural scattering should be eliminated. In Fig. 13, we indicate the results of the coverage-corrected elastic $t^{\prime}$-dependent and $N$-dependent extrapolations. Intercept values of Table I used in this comparison were normalized to constant electron polarization as discussed for Fig. 7. The $A$ vs $N$ and exponential fits reasonably follow $S$, a result partially anticipated in the earlier discussion. We thus feel confident that residual effects due to plural clastic scattering are eliminated by these two fitting forms, even when first-order scattering conditions cannot be guaranteed.

d. High precision measurements. The fact that morphology and fitting form are statistically unimportant to the intercept result in the $E=100 \mathrm{keV}$ elastic data led us to attempt to make a highly precise measurement of the intercept. This called for better count rates, which we obtained by increasing $\Delta E$ to $100 \mathrm{eV}$. Since curve 3 is flat over a $\Delta E$ range of $1300 \mathrm{eV}$ at $100 \mathrm{keV}$, this leads to negligible systematic error in the $t=0$ intercept. The result of $A$ vs $N$ for this experiment, taken over $8 \mathrm{~h}$ of running time, is shown in Fig. 14, and the fit results are given in Table I. For completeness, $A$ vs $N$ fits are shown for the elastic data as well. A disadvantage of $N$ fits is apparent in this figure: while $N$ values are easier to determine and have a smaller percentage error than do the corresponding thicknesses, they tend to be removed from the $N=0$ axis, especially at the lower energies, resulting in somewhat larger intercept uncertainties than those obtained from the $t$ fits (see Table I). Coverage corrections in the $E=100 \mathrm{keV}, \Delta E=100 \mathrm{eV}$ case eliminate the residual, statistically insignificant difference between the linear $t$ and $N$ fits, and indicate that even with the severe nonuniformity of our targets, they are so thin that plural scattering effects associated with varying morphology are important at a level below that of the difference between the $t$ and $t^{\prime}$ fits, i.e., $\sim 0.6 \%$. The overall precision of the intercept is $0.8 \%$, roughly a factor of 3 better than that quoted by Fletcher et al. ${ }^{15}$ It is expccted that this precision can be straightforwardly reduced by a factor of 2, which would make it comparable to accuracies quoted in the extremely careful double scattering measurements of Gellrich and Kessler. ${ }^{5}$ 


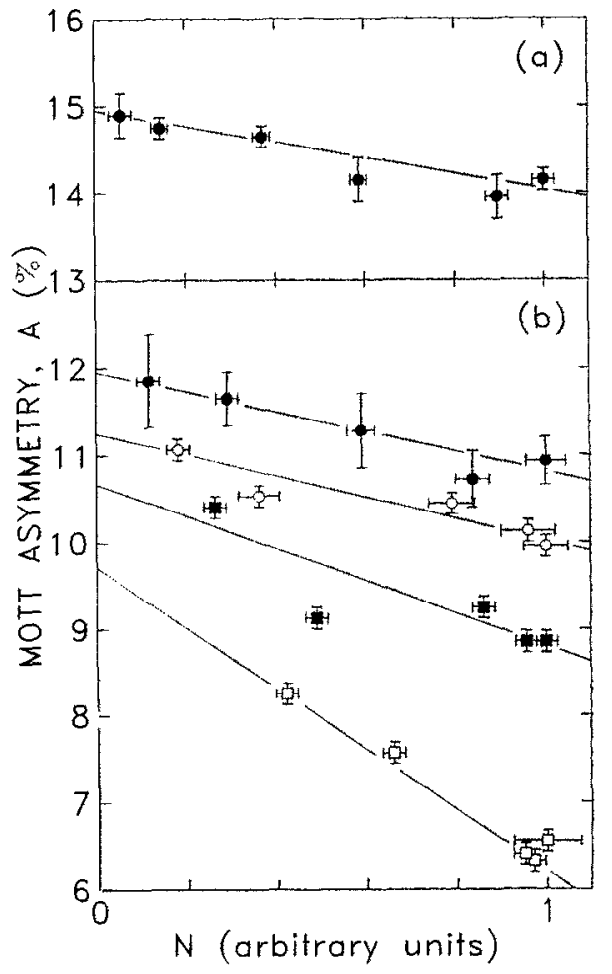

FIG. 14. Mott asymmetry vs total scattered count rate: (a) $E=100 \mathrm{keV}$, $\Delta E=100 \mathrm{eV}$-the two data points at right were taken with the thickest target; (b) elastic scattering data $(\Delta E=4 \mathrm{eV})$ taken between 20 and 100 $\mathrm{keV}$. Legend as in Fig. 10. The individual data sets are not normalized to constant electron polarization; the maximum $N$ for a given set is normalized to unity. Least-squares fits are indicated by the solid lines.

\section{CONCLUSIONS: RECOMMENDED EXTRAPOLATION PROCEDURES}

Deviations from $A_{\text {TRUE }}$ in the $(\Delta E, t)$ plane of Fig. 9 are essentially due to plural elastic scattering compounded with small-angle inelastic multiple scattering, which does little to further alter $A$. The probability of detecting plurally scattered electrons increases either with actual target thickness (curves 1 and 2), or "effective" target thickness, determined in turn by the value of $\Delta E$ (curves 3 and 4 ). Because elastic plural scattering is significantly more effective in reducing $A$ than is inelastic scattering associated with energy losses $\langle E$, curve 1 will generally exhibit appreciable deviations from $A_{\text {TRUE, }}$ as we have shown in these experiments, whereas curve 5 can be regarded for all intents and purposes as being independent of $\Delta E$. Thus, the appropriate procedure for obtaining $A_{\text {TRUE }}$ is a thickness extrapolation experiment at any $\Delta E$ value with a foil thickness range such that $A$ vs $t$ is demonstrably linear [i.e., the $A(t=0)$ value is independent of fitting function]. While large values of $\Delta E$ are advantageous in terms of count rate, and are unavoidable with standard Mott polarimeters, they require thinner foils to insure linearity. If experiments must be done at energies where the linear condition cannot be met, fitting of the data to the functional form $A=a$ $+b N$ or $A=a+b e^{-\lambda t}$ is recommended.

For high precision measurements, it is advisable to develop a plot similar to that shown in Fig. 9 so that the systematics of the analyzer being used can be understood thoroughly. This dictates the use of a Farago/Rice polarimeter, which we recommend strongly if high efficiency is not an issue, but accuracy and precision are important. Once calibration measurements including a thickness extrapolation and a careful measurement of curve 3 have been made, high accuracy measurements can be carried out with relatively large $\Delta E$ values and thick targets. In all cases, measurements should be made at as high an energy as possible to minimize systematic extrapolation errors, although practical limits on statistical uncertainties may dictate a compromise to lower energies.

\section{ACKNOWLEDGMENTS}

The authors wish to thank Professor M. Fink for numerous useful discussions, and C. McWhorter for careful instrument making. Several undergraduate assistants also contributed significantly to this research, including $T$. Harrison, D. Crutcher, C. Thornton, and M. Sieger. This work was supported by NSF grant number PHY-900772.

\section{APPENDIX: ENERGY-LOSS EXTRAPOLATIONS WITH IMPERFECT ANALYZER/DETECTORS}

In this appendix, the effects of finite energy resolution $(W>0)$ and/or the variation of electron detection efficiency with electron energy loss on the intercepts in $\Delta E$ extrapolations are discussed in detail. This is done in the context of a reanalysis of the data of Campbell et al., ${ }^{10}$ hereafter referred to as Campbell. We assume, as does Campbell, that the intensity of electrons impinging on their detector is independent of the electron energy loss $\epsilon$, i.e., $d N / d \epsilon$ is constant. The asymmetry $A$ associated with electrons of energy loss $\epsilon$ is taken as $A=A_{0}(1-\alpha \epsilon)$, where $\alpha$ is a constant and $A_{0}$ is the asymmetry for elastic scattering from the target. (Note that $A_{0}$ is not generally equal to $A_{\text {TRUE }}$, and that $\alpha$ is not the slope of $A$ vs $\Delta E$ ). Finally, the

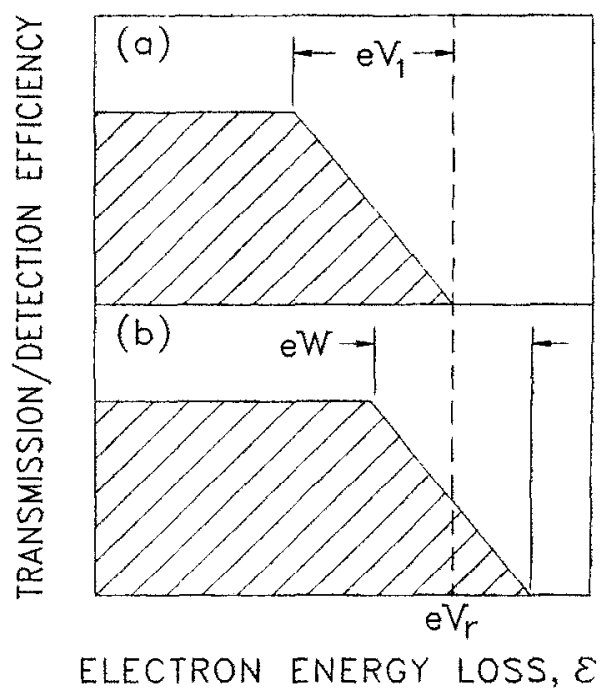

FIG. 15. Schematic plots of transmission/detection efficiency vs electron energy loss for (a), analyzers of the type described in Refs. 10 and 14 and (b), analyzers of the type described in this work and that of Ref. 7. 


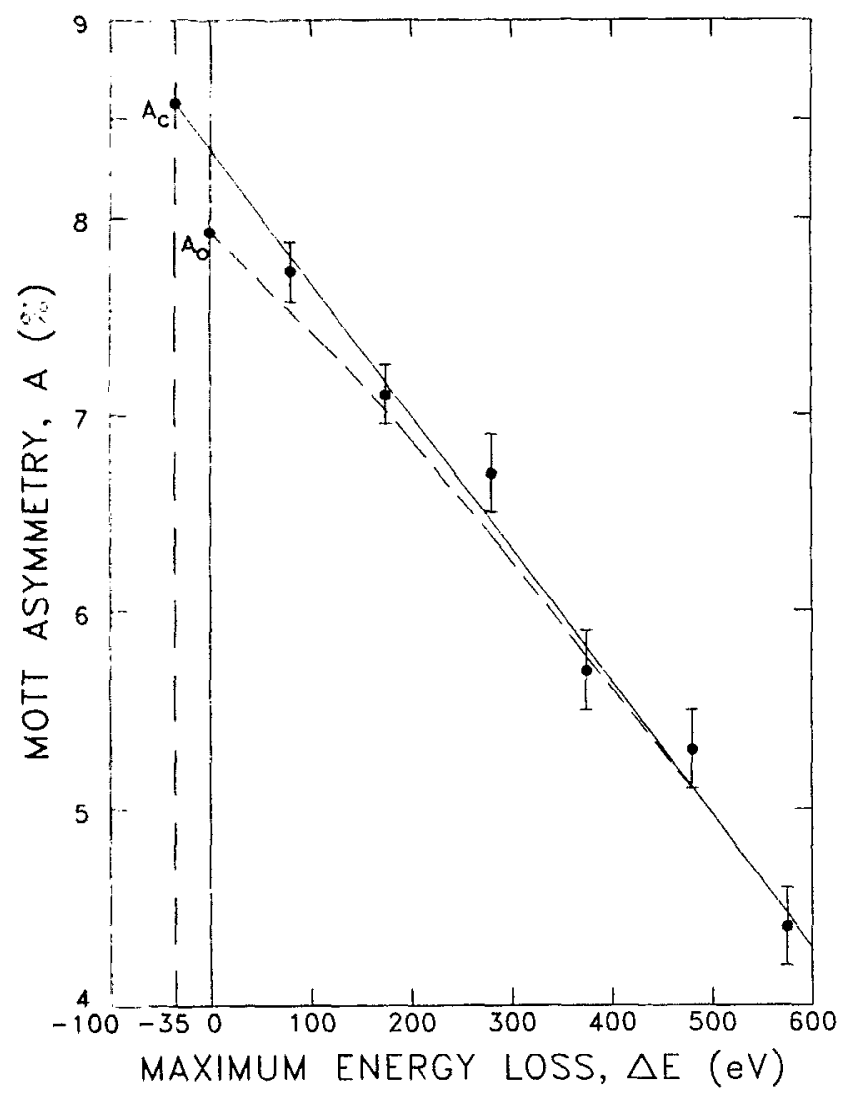

FIG. 16. Data of Campbell et al. (Ref. 10) taken at $E=30 \mathrm{keV}, t=300$ $\AA$ (see text).

detection efficiency is assumed constant until some value of electron energy loss $\epsilon=e V_{r}-e V_{1}\left(V_{r}=V_{b}\right.$ in Campbell's notation), at which point it drops linearly to $\epsilon=e V_{r}$ [see Fig. 15(a)]. In the detectors used by Campbell the CDEM cone itself is biased at $V_{r}$ so the response function is sharply cut off at $\epsilon=e V_{n}$ in contrast to our analyzer, whose response falls over the energy resolution width $W$, and which passes some electrons with $\epsilon$ 's greater than $V_{r}$ [see Fig. 15(b)]. Generally the response function of an RFA/electron multiplier combination is determined both by the multiplier detection efficiency and the transmission profile of the analyzer. Our apparatus and that of Campbell represent opposite ends of the range of possibilities in this regard: our response function is determined almost exclusively by the transmission characteristics of our RFA with $W \approx 4 \mathrm{eV}$, while theirs is essentially the detection efficiency curve of a standard CDEM, having $V_{1} \approx 70 \mathrm{eV}$, in good agreement with the measurements of, e.g., Bordoni. ${ }^{35}$

While the above assumptions are only approximately correct, they miss none of the important physics. The response curve shown in Fig. 15(a) is taken from Fig. 8(a) of Campbell, which, in combination with the assumption that $d N / d \epsilon$ is constant, yields the form of $N$ vs $V_{r}$ they observe. The experimental dependence of $A$ on $V_{r}$ should thus be

$$
A\left(V_{r}\right)=\int_{0}^{V_{r}} D(\epsilon) A(\epsilon) d \epsilon / \int_{0}^{V_{r}} D(\epsilon) d \epsilon,
$$

where $D(\epsilon)$ is the response function shown in $15(\mathrm{a})$. This expression can be evaluated for two cases: when $V_{r} \leqslant V_{1}$, and when $V_{r}>V_{1}$. In the former case, one obtains

$$
A\left(V_{r}\right)=A_{0}\left(1-\frac{1}{3} \alpha V_{r}\right),
$$

while in the latter case,

$$
A\left(V_{r}\right)=A_{0}\left[1-\frac{1}{3} \alpha\left(\frac{3 V_{r}^{2}+V_{1}^{2}-3 V_{r} V_{1}}{2 V_{r}-V_{1}}\right)\right] \text {. }
$$

For values of $V$,much larger than $V_{1}$, Eq. (A3) reduces to $A\left(V_{r}\right)=\left[1-(\alpha / 2) V_{r}\right]$, while for all $V_{r}$ below $V_{1}, A$ has a constant slope of $\alpha / 3$.

Figure 16 shows the $30-\mathrm{keV}$ data of Campbell, with both a linear least-squares fit and the function corresponding to Eqs. (A2) and (A3). The latter curve depends on the unknown quantities $\alpha$ and $A_{0}$. We have taken $\alpha / 2$ to be the slope of the linear fit, which corresponds to the high $V_{r}$ limit of Eq. (A3). In that the two curves converge for $V_{r}>400 \mathrm{~V}$, this is justified. The value of $A_{0}$ is then obtained by normalizing Eq. (A3) to the linear fit value at the highest experimental value of $V_{r}: 575 \mathrm{~V}$. Campbell quotes asymmetry values corresponding to the point $A_{c}$ in the figure, although we can find no justification for this. For response functions of the type shown in Fig. 15(b), such as ours, the appropriate procedure is to extrapolate the $A$ vs $V_{r}$ data to a point along the dashed line (extended to negative values of $V_{r}$ ) corresponding to $-W / 2$, as mentioned in the main text. (We speculate that this may have been the idea behind Campbell's analysis procedure.)

We have corrected the data in Fig. 9 of Campbell by multiplying their $S$ values by $\left(A_{0} / A_{c}\right)$, determined at each energy using the procedure described above and the slopes quoted in their Fig. 7. The correction factors, normalized to that at $100 \mathrm{keV}$, were $0.99,0.98,0.97,0.93$, and 0.90 for the data at $70,50,40,30$, and $20 \mathrm{keV}$, respectively. These modified data are shown in Fig. 13. While corrections of the same type would apply to any $\Delta E$ extrapolation, it is the large value of $V_{1}(70 \mathrm{~V})$ which makes the corrections as large as $10 \%$ in Campbell's case. With $W<10 \mathrm{~V}$, (e.g., for our analyzer and that of Uhrig et al. ${ }^{10}$ ), such corrections are completely negligible for all but the highest precision experiments.

${ }^{1}$ J. Kessler, Polarized Electrons, 2nd ed. (Springer, Berlin, 1985).

${ }^{2}$ D. T. Pierce, R. J. Celotta, M. H. Kelley, and J. Unguris, Nucl. Instrum. Methods A 266, 550 (1988).

${ }^{3}$ T. J. Gay and F. B. Dunning, Rev.Sci. Instrum. (in press).

${ }^{4}$ J. van Klinken, Nucl. Phys. 75, 161 (1966).

${ }^{5}$ A. Gellrich, K. Jost, and J. Kessler, Rev. Sci. Instrum. 61, 3399 (1990); A. Gellrich and J. Kessler, Phys. Rev. A 43, 204 (1991).

${ }^{6}$ D. M. Oro, W. H. Butler, F.-C. Tang, G. K. Walters, and F. B. Dunning, Rev. Sci. Instrum. 62, 667 (1991).

${ }^{7}$ M. Uhrig, A. Beck, J. Goeke, F. Eschen, M. Sohn, G. F. Hanne, K. Jost, and J. Kessler, Rev. Sci. Instrum. 60, 872 (1989).

${ }^{8}$ H. Hopster and D. L. Abraham, Rev. Sci. Instrum. 59, 49 (1988).

${ }^{9}$ H. Bienlein, G. Felsner, R. Fleischmann, K. Günther, H. von Issendorff, and H. Wegener, Z. Phys. 155, 101 (1959).

${ }^{10}$ D. M. Campbell, C. Hermann, G. Lampel, and R. Owen, J. Phys. E 18, 663 (1985).

${ }^{11}$ L. A. Hodge, T. J. Moravec, F. B. Dunning, and G. K. Walters, Rev. Sci. Instrum. 50, 5 (1979).

${ }^{12}$ L. G. Gray, M. W. Hart, F. B. Dunning, and G. K. Walters, Rev. Sci. Instrum. 55, 88 (1984). 
${ }^{13}$ F. B. Dunning, L. H. Gray, J. M. Ratliff, F.-C. Tang, X. Zhang, and G. K. Walters, Rev, Sci. Instrum. 58, 1706 (1987).

${ }^{14}$ J. J. McClelland, M. R. Scheinfein, and D. T. Pierce, Rev. Sci. Instrum. 60, 683 (1989).

${ }^{15}$ G. D. Fletcher, T. J. Gay, and M. S. Lubell, Phys. Rev. A 34, 911 (1986).

${ }^{16}$ F.-C. Tang, M. S. Lubell, K. Rubin, A. Vasilakis, M. Eminyan, and J. Slevin, Rev. Sci. Instrum. 57, 3004 (1986).

${ }^{17}$ X. Q. Guo, D. M. Crowe, M. S. Lubell, F.-C. Tang, A. Vasilakis, M. Eminyan, and J. Slevin, Rev. Sci. Instrum. 7, 1858 (1990).

${ }^{18}$ D. T. Pierce, R. J. Celotta, G.-C. Wang, W. N. Unertl, A. Galejs, C. E. Kuyatt, and S. R. Mielczarek, Rev. Sci. Instrum. 51, 478 (1980).

${ }^{19}$ Supplied by Crystal Specialties. Inc. with $\mathrm{Zn}$ doping of between 1 and $3 \times 10^{19} \mathrm{~cm}^{-3}$ and an etch pit density of 6500 .

${ }^{20}$ We found that the ability to precisely and reproducibly position the GaAs crystal under the $90^{\circ}$ hemispherical bend was important for obtaining maximum transmitted beam currents. Crystal positioning was accomplished with Vacuum Generators' RD7S (rotary motion) and LTM 100 (linear motion) feedthroughs.

${ }^{21}$ Maximum heating level was held for $20 \mathrm{~min}$ and was determined by visual estimation of the GaAs temperature.

${ }^{22}$ Spectra Diode Laboratories model SDL-2422-H1.

${ }^{23}$ D. T. Pierce and F. Meier, Phys. Rev. B 13, 5484 (1976).

${ }^{24}$ SAES Getters Inc. model CS/NF/3.9/12-T $14+14$ operated continuously at $3.8 \mathrm{~A}$.

${ }^{25}$ Monsanto 7/95E low molecular weight Formvar.

${ }^{26}$ P. E. Spivak, L. A. Mikaelyan, I. E. Kutikov, V. F. Apalin, I. I. Lukashevich, and G. V. Smirnov, Sov. Phys. JETP 14, 759 (1962).

${ }^{27}$ T. Nakanishi, K. Dohmae, S. Fukui, Y. Hayashi, I. Hirose, N. Horikawa, T. Ikoma, Y. Kamiya, M. Kurashina, and S. Okumi, Jpn. J. Appl. Phys. 25, 766 (1986).

${ }^{28}$ L. L. Kazmerski and D. M. Racine, J. Appl. Phys. 46, 791 (1975).

${ }^{29}$ K. L. Chopra, Thin Film Phenomena (McGraw-Hill, New York, 1969).

${ }^{30}$ V. A. A palin, I. Ye. Kutikov, I. I. Lukashevich, L. A. Mikaelyan, G. V. Smirnov, and P. Ye. Spivak, Nucl. Phys. 31, 657 (1962).

${ }^{31}$ H. Boersch, R. Schliepe, and K. E. Schriefl, Nucl. Phys. A 163, 625
(1971); H. Boersch, R. Wolter, and H. Schoenebeck, Z. Phys. 199, 124 (1967).

${ }^{32}$ J. L. Robins, Proc. Phys. Soc. (London) 78, 1177 (1962),

${ }^{33}$ J. A. Simpson, Rev. Sci. Instrum. 32, 1283 (1961).

${ }^{34}$ In experiments conducted by one of us (FBD) at Rice University, measured asymmetries actually decreased by several percent with the placement of transparent mesh in the RFAs. This effect is possibly due to reacceleration of secondary electrons emitted from the mesh into the target where they scatter by $60^{\circ}$ into the opposite detector.

${ }^{35} \mathrm{~F}$. Bordoni, Nucl. Instrum. Methods 97, 405 (1971).

${ }^{36}$ P. C. Gibbons, J. J. Ritsko, and S. E. Schnatterly, Rev. Sci. Instrum. 46, 1546 (1975).

${ }^{37}$ J. T. Park and F. D. Schowengerdt. Rev. Sci. Instrum. 40, 753 (1969).

${ }^{38}$ M. Fink (private communication).

${ }^{39}$ F. B. Dunning, F.CC. Tang, and G. K. Walters, Rev. Sci. Instrum. 58, 2195 (1987).

${ }^{40}$ O. C. Wells, Scanning Electron Microscopy (McGraw-Hill, New York, 1974), Chap. 3; D. L. Misell, Advan. Electron. Electron Phys. 32, 63 (1973).

${ }^{41}$ R. E. Burge and G. H. Smith, Proc. Phys. Soc. (London) 79, 673 (1962).

${ }^{42}$ M. R. Sogard, J. Appl. Phys. 51, 4417 (1980), and references therein.

${ }^{43}$ See, e.g., B. D. Gracher, S. S. Koslovskii, Yu. S. Korobochko, V. I. Mineev, and A. F. Petrochenko, Sov. Phys. JETP 52, 827 (1980).

${ }^{44}$ H. Wegener, Z. Phys. 151, $252(1958) ;$ M. E. Rose and H. A. Bethe, Phys. Rev. 55, 277 (1939).

${ }^{45}$ V. Hnizdo, Nucl. Instrum. Methods 109, 503 (1973).

to J. S. Greenberg, D. P. Malone, R. L. Gluckstern, and V. W. Hughes, Phys. Rev. 120, 1393 (1960).

${ }^{47}$ Philip R. Bevington, Data Reduction and Error Analysis for the Physical Sciences (McGraw-Hill, New York, 1969).

A. R. Brosi, A. I. Galonsky, B. H. Ketelle, and H. B. Willard, Nucl. Phys. 33, 353 (1962).

${ }^{49}$ R. F. Pinizotto (private communication).

${ }^{50}$ The percentage relative uncertainties in the $t$ values were assigned to the corresponding values of $t$.

${ }^{51}$ A. W. Ross and M. Fink, Phys. Rev. A 38, 6055 (1988). 